\title{
Regularity of traveling periodic stratified water waves with vorticity
}

\author{
Ling-Jun Wang* \\ School of Science, Wuhan University of Science and Technology, 430065 Wuhan, China
}

\begin{abstract}
We prove real analyticity of all the streamlines, including the free surface, of a steady stratified flow of water over a flat bed in the absence of stagnation points, with a Hölder continuous Bernoulli function and a Hölder continuously differentiable density function. Furthermore, we show that if the Bernoulli function and the density function possess some Gevrey regularity of index s, then the stream function admits the same Gevrey regularity throughout the fluid domain; in particular if the Gevrey index s equals to 1, then we obtain analyticity of the stream function. The regularity results hold for three distinct physical regimes: capillary, capillary-gravity, and gravity water waves.
\end{abstract}

Keywords: Analyticity, Gevrey regularity, wave profile, stratified water wave, vorticity

2010 MSC: 35B65, 35Q35, 76B03, 76B15

\section{Introduction}

We study regularity of the streamlines, including the surface profile, and regularity of the stream function, of stratified flows. Stratified flows are heterogeneous flows where the density varies as a function of the streamlines [17, 21, 24]. Stratification is a physically significant phenomenon for certain flows, where the fluid density may be caused to fluctuate by numerous factors such as the interplay between gravity and the salinity of the water. Recently, in [22, 23], the author developed an existence theory for two-dimensional stratified steady and periodic gravity waves, with or without surface tension. Using local and global bifurcation techniques, it was shown that, for stratified flows without stagnation points, there exist both small and large amplitude traveling periodic waves. Even more recently, some results ensuring the local existence of stratified waves, allowing for both surface tension and stagnation points, were proven by the authors in [12], extending the work of [6]. However, in the analysis of this paper, we will invoke the assumption of there being no stagnation points.

For homogeneous water waves, that is, waves with constant density, a series of works analyze the a priori regularity of the streamlines, and particularly the free-surface. For instance in the irrotational setting Lewy [15] showed that irrotational waves without stagnation points have real analytic profiles. Recent developments, proving the regularity of streamlines for rotational flows, were initiated by Constantin and Escher in [3] in the setting of homogeneous gravity waves over

\footnotetext{
${ }^{*}$ Corresponding author. Tel: +86 18062409592. Fax: +86 2768893261.

Email address: wanglingjun@wust.edu.cn (Ling-Jun Wang )
} 
a flat bed. Under the assumption that the vorticity function is Hölder continuously differentiable, it was shown in [3] that, each streamline, except the free surface, is real analytic; if further the vorticity function is real analytic, then the free surface itself is also analytic. The arguments in [3] base on translational invariance property of the resulting elliptic operator in the direction of wave propagation, and the celebrated result due to Kinderlehrer et al. 14] on regularity for elliptic free boundary problems. Later on, similar strong a priori regularity was established for a wide variety of homogeneous regimes, see for instance [9, 10, 11] for periodic gravity waves with surface tension, [19] for deep-water waves, [18] for flows with merely bounded vorticity, [8] for solitary-water waves, and the survey article [5]. In all the aforementioned works the analyticity of free surface is established under the extra assumption that the vorticity function is analytic. Quite recently, a similar result was established in [16] without the analyticity assumption on the vorticity function. Precisely, the authors in [16] showed that if the vorticity function is only Höder continuous, all the streamlines, including the free surface, of the steady homogeneous flow over a flat bed in the absence of stagnation points, are real analytic. The conclusions in [16] were achieved by using some a priori Schauder estimates and giving successively a quantitative bound for each derivative of the streamlines in the Höder norm. Moreover, studied also in [16] was the case when the vorticity possesses more regularity property rather than Höder continuity, namely Gevrey regularity of index $s$. Gevrey class is an intermediate space between the spaces of smooth functions and analytic functions, and the Gevrey class function of index 1 is just the real-analytic function; see Subsection 2.2 below for precise definition of Gevrey class. It was shown in [16] that if the vorticity is Gevrey regular, the stream function admits the same Gevrey regularity in the fluid domain, up to the free surface.

For heterogeneous, or stratified, water waves, some regularity results of steady periodic waves without stagnation points, have been studied recently in [13], in three distinct physical regimes, namely: capillary, capillary-gravity, and gravity water waves. There the authors proved, for all three types of waves, that, when the Bernoulli function is Höder continuous and the variable density function has a first derivative which is Höder continuous, then the free-surface profile is the graph of a smooth function. Furthermore, they showed that the streamlines are analytic a priori for capillary stratified waves, whereas for gravity and capillary-gravity stratified waves the streamlines are smooth in general, and analytic in an unstable regime; moreover, if the Bernoulli function and the streamline density function are both real analytic functions then all of the streamlines, including the wave profile, are real analytic for all gravity, capillary, and capillary-gravity stratified waves.

In the present work we follow the arguments in [16] for homogeneous water waves to study the regularity of the streamlines, including the surface profile, for stratified flows. We show, for the above three types of waves, that: 1) if the Bernoulli function is Höder continuous and the density function is Höder continuously differentiable, then all the streamlines, including the wave profile, are real analytic; see Theorem 2.2, 2) if both the Bernoulli function and the density function are in Gevrey (analytic) class, then the stream function admits the same Gevrey (analytic) regularity in the fluid domain, up to the free surface; see Theorem 2.3 stated in Subsection 2.2.

The paper is organized as follows. In Section 2 we formulate the rotational capillary-gravity stratified water-wave problem as free boundary problem for stream function and its equivalent reformulation in a fixed rectangular domain, and state our main regularity results. Notations and some useful inequalities are listed. Section 3 is devoted to the proof of analyticity of streamlines including the free surface. In Section 4 we study the Gevrey (analytic) regularity of stream function. 
In the last section, Section 5, we consider the traveling gravity water waves, and obtain similar regularity results for streamlines and stream function.

\section{Preliminaries and the main results}

\subsection{The governing equations for stratified water waves}

Consider a steady two-dimensional flow of an incompressible inviscid fluid with variable density and a steady wave on the free surface of the flow. By steady, we mean that the flow and the surface wave move at a constant speed from left to right without changing their configuration; that is, the velocity field of flow and the surface wave exhibit an $(t, X)$-dependence in the form of $X-c t$, where $X$ is the horizontal space variable, $c>0$ is the speed of the wave and $t$ denotes the time. Setting $x=X-c t$, we eliminate the time dependence of fluid flow and pass to the frame of reference moving with the wave. Assume that the flow is over a flat bed $y=-d$ with $0<d<\infty$, the free surface is given by $y=\eta(x)$ which is oscillating around the line $y=0$, and the liquid occupy the stationary domain

$$
\Omega=\left\{(x, y) \in \mathbb{R}^{2}:-d<y<\eta(x)\right\} .
$$

Also, the flow is assumed to be driven by capillarity (that is, surface tension) on the surface and gravity acting on the body of the fluid.

Let $u=u(x, y)$ and $v=v(x, y)$ denote the horizontal and vertical velocities, respectively, and let $\tilde{\rho}=\tilde{\rho}(x, y)>0$ be the density. Define the (relative) pseudo-stream function $\psi(x, y)$ by

$$
\psi_{y}=\sqrt{\tilde{\rho}}(u-c), \quad \psi_{x}=-\sqrt{\tilde{\rho}} v .
$$

The level sets $\{(x, y): \psi(x, y)=$ constant $\}$ are called streamlines of the fluid motion. The above relation (11) determines $\psi$ up to a constant. For definiteness we choose $\psi=0$ on the free boundary, so that $\psi=-p_{0}$ on $y=-d$, where $p_{0}<0$ is the (relative) pseudo-voumetric mass flux:

$$
p_{0}=\int_{-d}^{\eta(x)} \psi_{y}(x, y) d y .
$$

Since $\rho$ is transported, it must be constant on the streamlines and hence, we may think of it as a function of $\psi$ and assume

$$
\tilde{\rho}(x, y)=\rho(-\psi(x, y)),
$$

where $\rho:\left[p_{0}, 0\right] \rightarrow \mathbb{R}^{+}$is referred as streamline density function. Finally, consider only the case where there are no stagnation points throughout the fluid domain, that is,

$$
\psi_{y}(x, y) \leq-\delta<0 \quad \text { in } \bar{\Omega}
$$

for some $\delta>0$. Then the governing equations for the capillary-gravity stratified water wave problem are formulated as

$$
\begin{array}{rc}
\triangle \psi-g y \rho^{\prime}(-\psi)=-\beta(-\psi), & (x, y) \in \Omega_{\eta}, \\
|\nabla \psi|^{2}+2 g(y+d) \rho-2 \sigma \frac{\eta_{x x}}{\left(1+\eta_{x}^{2}\right)^{\frac{3}{2}}}=Q, & y=\eta(x), \\
\psi=0, & y=\eta(x), \\
\psi=-p_{0}, & y=-d .
\end{array}
$$


Here $\beta:\left[p_{0}, 0\right] \rightarrow \mathbb{R}$ is the Bernoulli function, $g>0$ is the gravitational constant of acceleration, $\sigma>0$ is the coefficient of surface tension, and $Q$ is a constant related to the energy. We refer to 23] for the detailed derivation of the above system of governing equations.

The equation (3a) is known as Yih's equation or the Yih-Long equation. The wave profile $\eta(x)$ represents an unknown in the problem since it is a free surface. Note that (3C) and (3d) imply that the free surface and flat bed are each level sets of $\psi$, and thus are streamlines. The system (3) with $g=0$ corresponds to the capillary stratified water waves.

\subsection{Statement of the main results}

To state our main results, we first recall the definition of Gevrey class functions, which is an intermediate space between the spaces of smooth functions and real-analytic functions; see [20] for more detail.

Definition 2.1. Let $W$ be an open subset of $\mathbb{R}^{d}$ and $f$ be a real-valued function defined on the closure $\bar{W}$ of $W$. We say $f$ belongs to Gevrey class in $\bar{W}$ of index $s \geq 1$, denoted by $f \in G^{s}(\bar{W})$, if $f \in C^{\infty}(\bar{W})$ and for any $x_{0} \in \bar{W}$ there exists a neighborhood $U$ of $x_{0}$ such that for any $x \in U \cap \bar{W}$, the series

$$
\sum_{\alpha \in \mathbb{N}^{d}} \frac{\partial^{\alpha} f\left(x_{0}\right)}{(\alpha !)^{s}}\left(x-x_{0}\right)^{\alpha}
$$

converges to $f(x)$.

Note that $f$ is real analytic in $\bar{W}$ if $s=1$. Observe $|\alpha| ! \leq 2^{|\alpha|} \alpha$ ! for any multi-index $\alpha \in \mathbb{N}^{d}$. Then as an alternative characterization for Gevrey class function, we have $f \in G^{s}(\bar{W})$ if for any compact subset $K \subset \bar{W}$, there exists a constant $C_{K}$, depending only on $K$, such that

$$
\forall \alpha \in \mathbb{N}^{d}, \quad \max _{x \in K}\left|\partial^{\alpha} f(x)\right| \leq C_{K}^{|\alpha|+1}(|\alpha| !)^{s} .
$$

In this paper we will derive a stronger estimate than the above one, namely,

$$
\forall \alpha \in \mathbb{N}^{d}, \quad \max _{x \in \bar{W}}\left|\partial^{\alpha} f(x)\right| \leq C^{|\alpha|+1}(|\alpha| !)^{s} .
$$

Throughout the paper let $C^{k, \mu}(\bar{W}), k \in \mathbb{N}, \mu \in(0,1)$, be the standard Hölder space of functions $f: \bar{W} \rightarrow \mathbb{R}$ with Hölder-continuous derivatives of exponent $\mu$ up to order $k$. For given $p_{0}<0, \beta \in C^{3, \mu}\left(\left[p_{0}, 0\right]\right)$ and $\rho \in C^{1, \mu}\left(\left[p_{0}, 0\right]\right)$, the existence of periodic water waves with or without surface tension has been established in [23, 22]. Our main result below shows that, with a Hölder continuous Bernoulli function and a Hölder continuously differentiable density function, each streamline can be described by the graph of some analytic function.

Theorem 2.2. Let $\psi(x, y)$ be the pseudo-stream function for the boundary problem (3a)-(3d) with free surface $y=\eta(x)$. Suppose $\beta \in C^{0, \mu}\left(\left[p_{0}, 0\right]\right)$ and $\rho \in C^{1, \mu}\left(\left[p_{0}, 0\right]\right)$ with $p_{0}<0$ and $0<\mu<1$ given. Then each streamline including the free surface $y=\eta(x)$ is a real-analytic curve.

The following result shows that the pseudo-stream function admits the same regularity as the vorticity. 
Theorem 2.3. Let $\psi(x, y)$ be the pseudo-stream function for the boundary problem (3a)-(3d) with free surface $y=\eta(x)$. Suppose $\beta, \rho \in G^{s}\left(\left[p_{0}, 0\right]\right)$ with $p_{0}<0$ and $s \geq 1$ given. Then $\psi(x, y) \in$ $G^{s}(\bar{\Omega})$; in particular if $s=1$, i.e., $\beta$ and $\rho$ are analytic in $\left[p_{0}, 0\right]$, then the pseudo-stream function $\psi(x, y)$ is analytic in $\bar{\Omega}$.

Remark 2.4. The above results also hold for the traveling gravity water waves; see Theorem 5.1 in Section 5. Moreover, although we focus on the capillary-gravity water waves in this and the next two subsequent sections, our arguments are also applicable for capillary waves, that is, waves driven only by the surface tension by setting $g=0$. Accordingly, the conclusions in these two sections also hold for the capillary waves.

\subsection{Reformulation}

Under the no-stagnation assumption (2), we can use the partial hodograph change of variables to transform the free boundary problem (3a $)-(3 \mathrm{~d} d)$ into a problem with fixed boundary. Precisely, if we introduce the new variable $(q, p)$ with

$$
q=x, \quad p=-\psi(x, y),
$$

and exchange the roles of the $y$-coordinate and $\psi$ by setting

$$
h(q, p)=y+d,
$$

then the fluid domain $\Omega$ is transformed into a fixed infinite strip

$$
R=\left\{(q, p): q \in \mathbb{R}, p_{0}<p<0\right\},
$$

and the system (3a)-(3d) can be reformulated in this strip as

$$
\begin{aligned}
\left(1+h_{q}^{2}\right) h_{p p}-2 h_{p} h_{q} h_{p q}+h_{p}^{2} h_{q q}-g(h-d) \rho_{p} h_{p}^{3}+\beta(p) h_{p}^{3}=0, & \text { in } R, \\
1+h_{q}^{2}+\left(2 g \rho h-2 \sigma \frac{h_{q q}}{\left(1+h_{q}^{2}\right)^{3 / 2}}-Q\right) h_{p}^{2}=0, & \text { on } p=0, \\
h=0, & \text { on } p=p_{0} .
\end{aligned}
$$

We refer to [22, 23] for the equivalence of the two systems (3a)-(3d) and (4a)-(4c) of governing equations. Note that $h_{p}=\frac{1}{c-u}$. The no-stagnation assumption (2) ensures that

$$
0<\inf _{(q, p) \in \bar{R}} h_{p} \leq h_{p} \leq \sup _{(q, p) \in \bar{R}} h_{p} \leq \frac{1}{\delta} .
$$

The following proposition shows that the regularity is preserved through hodograph transformation. So we only need to study the above problem (4a)-(4c) instead of the original one (3a) $-(3 \mathrm{~d})$.

Proposition 2.5. Let $h \in C^{2, \mu}(\bar{R})$ be a solution to the problem (4a)-(4C) . If the mapping $q \mapsto$ $h(q, p)$, with any fixed $p \in\left[p_{0}, 0\right]$, is analytic in $\mathbb{R}$, then each streamline including the free surface is an analytic curve. Moreover if $h \in G^{s}(\bar{R})$ then the pseudo-stream function $\psi$ for (3a)-(3d) lies in $G^{s}(\bar{\Omega})$; in particular $\psi$ is analytic in $\bar{\Omega}$ provided $h$ is analytic in $\bar{R}$.

We refer to [16] for the proof of the above proposition, which is an application of [2, Theorem 3.1]. Some of the technical results in the present paper are similar to those in [16], to which we refer for their proofs. 


\subsection{Notations and some useful inequalities}

We list some notations and useful inequalities which will be used throughout the paper. Let $k \in \mathbb{N}$ and $\mu \in(0,1)$, and let $\left(C^{k, \mu}(\bar{R}) ;\|\cdot\|_{k, \mu ; \bar{R}}\right)$ be the standard Hölder space equipped with the norm

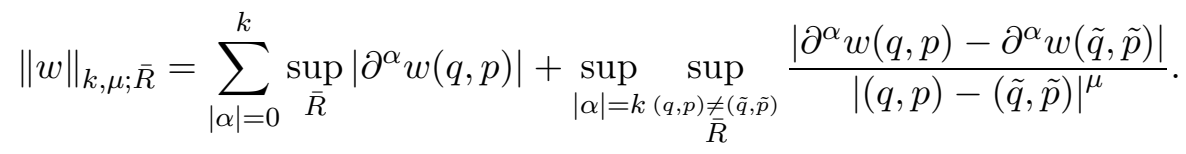

To simplify the notation we will use the notation $\|\cdot\|_{k, \mu}$ instead of $\|\cdot\|_{k, \mu ; \bar{R}}$ if no confusion occurs. For the case when $\mu=0$, we naturally define

$$
\|w\|_{k}=\|w\|_{k, 0}=\sum_{|\alpha|=0}^{k} \sup _{\bar{R}}\left|\partial^{\alpha} w\right|
$$

For $\mu \in(0,1)$, direct verification shows that

$$
\|u w\|_{0, \mu} \leq\|u\|_{0, \mu}\|w\|_{0, \mu}, \quad\|u w\|_{1, \mu} \leq 2\|u\|_{1, \mu}\|w\|_{1, \mu} .
$$

For a multi-index $\alpha=\left(\alpha_{1}, \alpha_{2}\right) \in \mathbb{N}^{2}$, we denote $\partial^{\alpha}=\partial_{q}^{\alpha_{1}} \partial_{p}^{\alpha_{2}}, \alpha !=\alpha_{1} ! \alpha_{2} !$ and denote the length of $\alpha$ by $|\alpha|=\alpha_{1}+\alpha_{2}$. Moreover for two multi-indices $\alpha$ and $\beta=\left(\beta_{1}, \beta_{2}\right) \in \mathbb{N}^{2}$, by $\beta \leq \alpha$ we mean $\beta_{i} \leq \alpha_{i}$ for each $1 \leq i \leq 2$. Let $\left(\begin{array}{l}\alpha \\ \beta\end{array}\right)$ be the binomial coefficient, i.e.,

$$
\left(\begin{array}{l}
\alpha \\
\beta
\end{array}\right)=\frac{\alpha !}{\beta !(\alpha-\beta) !}=\frac{\alpha_{1} ! \alpha_{2} !}{\beta_{1} !\left(\alpha_{1}-\beta_{1}\right) ! \beta_{2} !\left(\alpha_{2}-\beta_{2}\right) !} .
$$

In the sequel, we use the convention that $m !=1$ if $m \leq 0$.

We now list without proof some straightforward inequalities to be used frequently later.

(i) For any $\beta \leq \alpha$, we have

$$
\left(\begin{array}{l}
\alpha \\
\beta
\end{array}\right)=\frac{\alpha !}{\beta !(\alpha-\beta) !} \leq \frac{|\alpha| !}{|\beta| !(|\alpha|-|\beta|) !}=\left(\begin{array}{c}
|\alpha| \\
|\beta|
\end{array}\right)
$$

(ii) Given $s \geq 1$, then $(m !)^{s-1}(n !)^{s-1} \leq[(m+n) !]^{s-1}$.

(iii) Given $m \geq 1$, we have, for any integer $k$ with $2 \leq k \leq 3$,

$$
\sum_{0<j<m} \frac{|m|^{k}}{|j|^{k}(m-j)^{k}} \leq 2^{k} \pi^{2}
$$


Lemma 2.6. Given $\alpha \in \mathbb{N}^{2}$, we have

$$
\begin{gathered}
\sum_{\beta \leq \alpha, 0<|\beta|<|\alpha|} \frac{|\alpha|^{2}}{|\beta|^{3}(|\alpha|-|\beta|)^{2}} \leq 8 \pi^{2}, \\
\sum_{\beta \leq \alpha, 0<|\beta|<|\alpha|} \frac{|\alpha|^{2}}{|\beta|^{2}(|\alpha|-|\beta|)^{2}} \leq 8 \pi^{2}|\alpha|, \\
\sum_{\beta \leq \alpha, 0<|\beta|<|\alpha|} \frac{|\alpha|}{|\beta|^{3}(|\alpha|-|\beta|)} \leq 8 \pi^{2}, \\
\sum_{\beta \leq \alpha, 0<|\beta|<|\alpha|} \frac{|\alpha|^{3}}{|\beta|^{4}(|\alpha|-|\beta|)^{3}} \leq 8 \pi^{2} .
\end{gathered}
$$

We refer to [16, Lemma 2.6] for the proof of the lemma.

\section{Analyticity of streamlines}

We prove now the analyticity of streamlines, including the free surface $y=\eta(x)$. In view of Proposition 2.5, it suffices to show the following proposition, which concludes that the map $q \mapsto h(q, p)$ is analytic for all $p \in\left[p_{0}, 0\right]$.

Proposition 3.1. Let $\beta \in C^{0, \mu}\left(\left[p_{0}, 0\right]\right)$ and $\rho \in C^{1, \mu}\left(\left[p_{0}, 0\right]\right)$ with $p_{0}<0$ and $0<\mu<1$ given, and $h \in C^{2, \mu}(\bar{R})$ be a solution of the governing equations (4a)-(4c). Then there exists a constant $L \geq 1$, such that for all $m \in \mathbb{N}$ with $m \geq 2$, one has

$$
\left(E_{m}\right): \quad\left\|\partial_{q}^{m} h\right\|_{2, \mu} \leq L^{m-1}(m-2) ! .
$$

Thus the map $q \mapsto h(q, p)$ is analytic for all $p \in\left[p_{0}, 0\right]$.

Remark 3.2. As to be seen in the proof below, the constant $L$ depends on $\mu, \sigma, \inf _{\bar{R}} h_{p},\|h\|_{2, \mu}$, $\|\beta\|_{0, \mu},\|\rho\|_{1, \mu}$ and the number $\delta$ given in (5), but independent of the order $m$ of derivative.

Remark 3.3. Starting from the $C^{2, \mu}$-regularity solution $h$ of the governing equations (4a)-(4c), we use the Schauder estimate ( cf. [7, Theorem 6.30]) for $\partial_{q} h$ which satisfies a nonlinear elliptic equation of the same type as (4a)-(4c), to conclude that $\partial_{q} h \in C^{2, \mu}(\bar{R})$. Repeating the procedure, we can derive by standard iteration that $\partial_{q}^{k} h \in C^{2, \mu}(\bar{R})$ for any $k \in \mathbb{N}$; see for instance [4, 13].

To confirm the last statement in the above proposition 3.1, we choose $C$ in such a way that

$$
C=\max \left\{L,\|h\|_{1, \mu}\right\},
$$

which, along with the estimate $\left(E_{m}\right)$ with $m \geq 2$ in Proposition [3.1, yields

$$
\forall m \in \mathbb{N}, \quad \max _{(q, p) \in \bar{R}}\left|\partial_{q}^{m} h(q, p)\right| \leq C^{m+1} m ! .
$$

In particular, for any $p \in\left[p_{0}, 0\right], \max _{q \in \mathbb{R}}\left|\partial_{q}^{m} h(q, p)\right| \leq C^{m+1} m$ !. This gives the real analyticity of the map $q \mapsto h(q, p), p \in\left[p_{0}, 0\right]$.

Before proving the above proposition, we first give the following technical lemma and refer to [16, Lemma 3.4] for its proof. 
Lemma 3.4. Let $\ell=1$ or 2 be given, and let $\|\cdot\|$ stand for the Hölder norm $\|\cdot\|_{0, \mu}$ or $\|\cdot\|_{1, \mu}$. Suppose that $k_{0}$ is an integer with $k_{0} \geq \ell+1$, and $\partial_{q}^{k} u_{j} \in C^{0, \mu}(\bar{R})$ for all $k \leq k_{0}, j=1,2,3$. If there exists a constant $H \geq 1$ such that

$$
\forall \ell+1 \leq k \leq k_{0}, \quad\left\|\partial_{q}^{k} u_{j}\right\| \leq H^{k-\ell}(k-\ell-1) !, \quad j=1,2,3,
$$

then we can find a constant $C_{*}$ depending only on $\ell$ such that

$$
\forall \ell+1 \leq k \leq k_{0}, \quad\left\|\partial_{q}^{k}\left(u_{1} u_{2} u_{3}\right)\right\| \leq C_{*}\left(\sum_{j=1}^{3}\left\|u_{j}\right\|_{\ell+1, \mu}+1\right)^{3} H^{k-\ell}(k-\ell-1) ! .
$$

We now prove Proposition 3.1 .

Proof of Proposition [3.1. In view of Remark 3.3 we may assume that $\partial_{q}^{k} h \in C^{2, \mu}(\bar{R})$ for any $k \in \mathbb{N}$. Now we prove the validity of $\left(E_{m}\right)$ by using induction on $m$. For $m=2,\left(E_{m}\right)$ obviously holds if we choose

$$
L \geq\left\|\partial_{q}^{2} h\right\|_{2, \mu}+1
$$

Now assume that $\left(E_{j}\right)$ holds for all $j \in \mathbb{N}$ with $2 \leq j \leq m-1$ and $m \geq 3$, that is,

$$
\left\|\partial_{q}^{j} h\right\|_{2, \mu} \leq L^{j-1}(j-2) !, \quad 2 \leq j \leq m-1 .
$$

Then we show the validity of $\left(E_{m}\right)$. For this purpose, taking the derivative with respect to $q$ up to order $m$ on both sides of equations (4a)-(4c), and then applying Leibniz formula, we have

$$
\begin{cases}A(h)\left[\partial_{q}^{m} h\right]=f_{1}+f_{2} & \text { in } R \\ B(h)\left[\partial_{q}^{m} h\right]=\varphi_{1}+\varphi_{2} & \text { on } p=0 \\ \partial_{q}^{m} h=0 & \text { on } p=p_{0}\end{cases}
$$

where the operators

$$
A(h)[\phi]=\left(1+h_{q}^{2}\right) \phi_{p p}-2 h_{q} h_{p} \phi_{q p}+h_{p}^{2} \phi_{q q}, \quad B(h)[\phi]=2 \sigma \frac{h_{p}^{2}}{\left(1+h_{q}^{2}\right)^{\frac{3}{2}}} \partial_{q}^{2}
$$

and the right-hand side

$$
\begin{aligned}
f_{1} & =\sum_{1 \leq n \leq m}\left(\begin{array}{c}
m \\
n
\end{array}\right)\left[-\left(\partial_{q}^{n} h_{q}^{2}\right)\left(\partial_{q}^{m-n} h_{p p}\right)+2\left(\partial_{q}^{n}\left(h_{p} h_{q}\right)\right)\left(\partial_{q}^{m-n} h_{p q}\right)-\left(\partial_{q}^{n} h_{p}^{2}\right)\left(\partial_{q}^{m-n} h_{q q}\right)\right] \\
f_{2} & =-\beta(p)\left(\partial_{q}^{m} h_{p}^{3}\right)+g \rho_{p} \sum_{0 \leq n \leq m}\left(\begin{array}{c}
m \\
n
\end{array}\right)\left(\partial_{q}^{n}(h-d)\right)\left(\partial_{q}^{m-n} h_{p}^{3}\right) \\
\varphi_{1} & =\partial_{q}^{m} h_{q}^{2}+2 g \rho \partial_{q}^{m}\left(h h_{p}^{2}\right)-Q\left(\partial_{q}^{m} h_{p}^{2}\right) \\
\varphi_{2} & =-2 \sigma \sum_{1 \leq n \leq m}\left(\partial_{q}^{m-n} h_{q q}\right)\left(\partial_{q}^{n} \frac{h_{p}^{2}}{\left(1+h_{q}^{2}\right)^{\frac{3}{2}}}\right) .
\end{aligned}
$$


The operator $A(h)$ is uniformly elliptic since its coefficients satisfy

$$
\left(1+h_{q}^{2}\right) h_{p}^{2}-h_{q}^{2} h_{p}^{2}=h_{p}^{2} \geq \inf _{\bar{R}} h_{p}^{2}>0
$$

due to (5). Moreover it has been shown in [10, 13] that the operator $B(h)$ satisfies the complementing condition in the sense of [1]. Since $h \in C^{2, \mu}(\bar{R})$ the coefficients of the operators $A(h)$ and $B(h)$ are in $C^{1, \mu}(\bar{R})$. Moreover, by virtue of the induction assumption (10), one has $\partial_{q}^{i} \partial_{p}^{j} h \in C^{0, \mu}(\bar{R})$ for all multi-index $(i, j)$ with $i+j \leq m+1$ and $j \leq 2$, and similarly $\partial_{q}^{i} \partial_{p}^{j} h \in C^{1, \mu}(\bar{R})$ for all multi-index $(i, j)$ with $i+j \leq m$ and $j \leq 1$. As a result, the right-hand side $f_{i}, \varphi_{i} \in C^{0, \mu}(\bar{R}), i=1,2$, since by (6) the product of two functions in $C^{k, \mu}(\bar{R})$ is still in $C^{k, \mu}(\bar{R})$ with $k=0,1$. Thus, the standard Schauder estimate (see for instance [1])

$$
\left\|\partial_{q}^{m} h\right\|_{2, \mu} \leq \mathcal{C}\left(\left\|\partial_{q}^{m} h\right\|_{0}+\sum_{i=1}^{2}\left\|f_{i}\right\|_{0, \mu}+\sum_{i=1}^{2}\left\|\varphi_{i}\right\|_{0, \mu}\right)
$$

holds, where $\mathcal{C}$ is a constant depending only on $\mu, \delta, \inf _{\bar{R}} h_{p}$ and $\|h\|_{2, \mu}$. To show $\left(E_{m}\right)$ is valid, we estimate the terms on the right-hand side of (16) through the following steps.

To simplify the notations, we will use $C_{j}, j \geq 1$, to denote suitable harmless constants larger than 1. By harmless constants we mean that they are independent of $m$.

Step 1) We claim that there exists $C_{1}>0$ such that, with $m \geq 3$,

$$
\left\|\partial_{q}^{m} h\right\|_{0} \leq C_{1} L^{m-2}(m-2) !
$$

Indeed, when $m=3$ the above estimate obviously holds if we choose $C_{1}=\|h\|_{3, \mu}+1$; when $m \geq 4$ it follows from the induction assumption (10) that

$$
\left\|\partial_{q}^{m} h\right\|_{0} \leq\left\|\partial_{q}^{m-2} h\right\|_{2, \mu} \leq L^{m-3}(m-4) ! \leq L^{m-2}(m-2) ! .
$$

Then (17) follows.

Step 2) Let $f_{1}$ be given in (12). In this step we prove

$$
\left\|f_{1}\right\|_{0, \mu} \leq C_{2} L^{m-2}(m-2) !
$$

Observe that, by (무),

$$
\begin{array}{r}
\left\|f_{1}\right\|_{0, \mu} \leq \sum_{1 \leq n \leq m}\left(\begin{array}{c}
m \\
n
\end{array}\right)\left\|\partial_{q}^{n} h_{q}^{2}\right\|_{0, \mu}\left\|\partial_{q}^{m-n} h_{p p}\right\|_{0, \mu}+2 \sum_{1 \leq n \leq m}\left(\begin{array}{c}
m \\
n
\end{array}\right)\left\|\partial_{q}^{n}\left(h_{p} h_{q}\right)\right\|_{0, \mu}\left\|\partial_{q}^{m-n} h_{p q}\right\|_{0, \mu} \\
+\sum_{1 \leq n \leq m}\left(\begin{array}{c}
m \\
n
\end{array}\right)\left\|\partial_{q}^{n} h_{p}^{2}\right\|_{0, \mu}\left\|\partial_{q}^{m-n} h_{q q}\right\|_{0, \mu} .
\end{array}
$$

We now treat the first term on the right-hand side, and write

$$
\begin{aligned}
& \sum_{1 \leq n \leq m}\left(\begin{array}{c}
m \\
n
\end{array}\right)\left\|\partial_{q}^{n} h_{q}^{2}\right\|_{0, \mu}\left\|\partial_{q}^{m-n} h_{p p}\right\|_{0, \mu} \leq \sum_{1 \leq n \leq m}\left(\begin{array}{c}
m \\
n
\end{array}\right)\left\|\partial_{q}^{n} h_{q}^{2}\right\|_{0, \mu}\left\|\partial_{q}^{m-n} h\right\|_{2, \mu} \\
& \leq\left(\sum_{1 \leq n \leq 2}+\sum_{3 \leq n \leq m-2}+\sum_{m-1 \leq n \leq m}\right)\left(\begin{array}{c}
m \\
n
\end{array}\right)\left\|\partial_{q}^{n} h_{q}^{2}\right\|_{0, \mu}\left\|\partial_{q}^{m-n} h\right\|_{2, \mu} .
\end{aligned}
$$


By the induction assumption (10), one has

$$
\forall 3 \leq n \leq m, \quad\left\|\partial_{q}^{n} h_{q}\right\|_{0, \mu} \leq\left\|\partial_{q}^{n-1} h\right\|_{2, \mu} \leq L^{n-2}(n-3) ! .
$$

Thus applying Lemma 3.4, with $\ell=2, k_{0}=m, H=L, u_{1}=u_{2}=h_{q}$ and $u_{3}=1$, yields that

$$
\forall 3 \leq n \leq m, \quad\left\|\partial_{q}^{n} h_{q}^{2}\right\|_{0, \mu} \leq C_{5} L^{n-2}(n-3) ! .
$$

Moreover, we have

$$
\forall 1 \leq n \leq m-2, \quad\left\|\partial_{q}^{m-n} h\right\|_{2, \mu} \leq L^{m-n-1}(m-n-2) !
$$

due to the induction assumption (10). Then using the above two estimates, straightforward verification shows that

$$
\sum_{1 \leq n \leq 2}\left(\begin{array}{c}
m \\
n
\end{array}\right)\left\|\partial_{q}^{n} h_{q}^{2}\right\|_{0, \mu}\left\|\partial_{q}^{m-n} h\right\|_{2, \mu}+\sum_{m-1 \leq n \leq m}\left(\begin{array}{c}
m \\
n
\end{array}\right)\left\|\partial_{q}^{n} h_{q}^{2}\right\|_{0, \mu}\left\|\partial_{q}^{m-n} h\right\|_{2, \mu} \leq C_{6} L^{m-2}(m-3) !
$$

if we choose

$$
C_{6} \geq\left(\|h\|_{3, \mu}+1\right)\left(30\|h\|_{3, \mu}+4 C_{5}+6\right) .
$$

Next for the case when $3 \leq n \leq m-2$, which appears only when $m \geq 5$, combination of the estimates (21) and (22) gives

$$
\begin{aligned}
\sum_{3 \leq n \leq m-2}\left(\begin{array}{c}
m \\
n
\end{array}\right)\left\|\partial_{q}^{n} h_{q}^{2}\right\|_{0, \mu}\left\|\partial_{q}^{m-n} h\right\|_{2, \mu} & \leq C_{5} \sum_{3 \leq n \leq m-2} \frac{m !}{n !(m-n) !} L^{n-2}(n-3) ! L^{m-n-1}(m-n-2) ! \\
& \leq C_{7} L^{m-3}(m-2) ! \sum_{3 \leq n \leq m-2} \frac{m^{2}}{n^{3}(m-n)^{2}} \\
& \leq C_{8} L^{m-3}(m-2) ! .
\end{aligned}
$$

This along with (23) shows, in view of (20),

$$
\sum_{1 \leq n \leq m}\left(\begin{array}{c}
m \\
n
\end{array}\right)\left\|\partial_{q}^{n} h_{q}^{2}\right\|_{0, \mu}\left\|\partial_{q}^{m-n} h_{p p}\right\|_{0, \mu} \leq\left(C_{6}+C_{8}\right) L^{m-2}(m-2) !
$$

Similarly, we can find a constant $C_{9}$ such that

$2 \sum_{1 \leq n \leq m}\left(\begin{array}{c}m \\ n\end{array}\right)\left\|\partial_{q}^{n}\left(h_{p} h_{q}\right)\right\|_{0, \mu}\left\|\partial_{q}^{m-n} h_{p q}\right\|_{0, \mu}+\sum_{1 \leq n \leq m}\left(\begin{array}{c}m \\ n\end{array}\right)\left\|\partial_{q}^{n} h_{p}^{2}\right\|_{0, \mu}\left\|\partial_{q}^{m-n} h_{q q}\right\|_{0, \mu} \leq C_{9} L^{m-2}(m-2) !$.

Inserting the above two estimates into (19), we get the desired estimate (18) by choosing $C_{2}=$ $C_{6}+C_{8}+C_{9}$.

Step 3) We now prove

$$
\left\|f_{2}\right\|_{0, \mu} \leq C_{3} L^{m-2}(m-2) !
$$

In fact, using (6) we have

$$
\left\|f_{2}\right\|_{0, \mu} \leq\|\beta\|_{0, \mu}\left\|\partial_{q}^{m} h_{p}^{3}\right\|_{0, \mu}+g\|\rho\|_{1, \mu} \sum_{0 \leq n \leq m}\left(\begin{array}{c}
m \\
n
\end{array}\right)\left\|\partial_{q}^{n}(h-d)\right\|_{0, \mu}\left\|\partial_{q}^{m-n} h_{p}^{3}\right\|_{0, \mu} .
$$


Next we estimate the two terms on the right-hand side.

By the induction assumption (10), one has

$$
\forall 3 \leq j \leq m, \quad\left\|\partial_{q}^{j} h_{p}\right\|_{0, \mu} \leq\left\|\partial_{q}^{j-1} h\right\|_{2, \mu} \leq L^{j-2}(j-3) ! .
$$

Then using Lemma 3.4, with $\ell=2, k_{0}=m, H=L, u_{1}=u_{2}=u_{3}=h_{p}$, we conclude

$$
\left\|\partial_{q}^{m} h_{p}^{3}\right\|_{0, \mu} \leq C_{10} L^{m-2}(m-3) !
$$

Similarly we have

$$
\forall 0 \leq n \leq m-3, \quad\left\|\partial_{q}^{m-n} h_{p}^{3}\right\|_{0, \mu} \leq C_{10} L^{m-n-2}(m-n-3) ! .
$$

Write

$$
\begin{aligned}
& \sum_{0 \leq n \leq m}\left(\begin{array}{c}
m \\
n
\end{array}\right)\left\|\partial_{q}^{n}(h-d)\right\|_{0, \mu}\left\|\partial_{q}^{m-n} h_{p}^{3}\right\|_{0, \mu} \\
= & \left(\sum_{0 \leq n \leq 3}+\sum_{4 \leq n \leq m-3}+\sum_{m-2 \leq n \leq m}\right)\left(\begin{array}{c}
m \\
n
\end{array}\right)\left\|\partial_{q}^{n}(h-d)\right\|_{0, \mu}\left\|\partial_{q}^{m-n} h_{p}^{3}\right\|_{0, \mu} .
\end{aligned}
$$

Using the induction assumption (10) and (27), we can compute directly to obtain

$$
\left(\sum_{0 \leq n \leq 3}+\sum_{m-2 \leq n \leq m}\right)\left\|\partial_{q}^{n}(h-d)\right\|_{0, \mu}\left\|\partial_{q}^{m-n} h_{p}^{3}\right\|_{0, \mu} \leq C_{11} L^{m-2}(m-3) !
$$

by choosing

$$
C_{11} \geq C_{10}\left(9\|h\|_{2, \mu}+d\right)+6\|h\|_{2, \mu}\left\|h_{p}^{3}\right\|_{2, \mu}+12\left\|h_{p}^{3}\right\|_{2, \mu} .
$$

For the case when $4 \leq n \leq m-3$, again by (10) and (27) we have

$$
\begin{aligned}
& \sum_{4 \leq n \leq m-3}\left(\begin{array}{c}
m \\
n
\end{array}\right)\left\|\partial_{q}^{n}(h-d)\right\|_{0, \mu}\left\|\partial_{q}^{m-n} h_{p}^{3}\right\|_{0, \mu} \\
\leq & \sum_{4 \leq n \leq m-3}\left(\begin{array}{c}
m \\
n
\end{array}\right)\left\|\partial_{q}^{n-2} h\right\|_{2, \mu}\left\|\partial_{q}^{m-n} h_{p}^{3}\right\|_{0, \mu} \\
\leq & \sum_{4 \leq n \leq m-3} \frac{m !}{n !(m-n) !} C_{10} L^{n-3}(n-4) ! L^{m-n-2}(m-n-3) ! \\
\leq & C_{12} L^{m-5}(m-3) ! \sum_{4 \leq n \leq m-3} \frac{m^{3}}{n^{4}(m-n)^{3}} \\
\leq & C_{13} L^{m-2}(m-3) ! .
\end{aligned}
$$

Inserting (29) and (30) into (28), we get

$$
\sum_{0 \leq n \leq m}\left(\begin{array}{c}
m \\
n
\end{array}\right)\left\|\partial_{q}^{n}(h-d)\right\|_{0, \mu}\left\|\partial_{q}^{m-n} h_{p}^{3}\right\|_{0, \mu} \leq\left(C_{11}+C_{13}\right) L^{m-2}(m-3) !
$$


Thus combining the above estimate and (26), we obtain, in view of (25), the desired estimate (24), by choosing $C_{3}=C_{10}\|\beta\|_{0, \mu}+g\|\rho\|_{1, \mu}\left(C_{11}+C_{13}\right)+1$.

Step 4) Finally we prove

$$
\sum_{i=1}^{2}\left\|\varphi_{i}\right\|_{0, \mu} \leq C_{4} L^{m-2}(m-2) ! .
$$

First for $\left\|\varphi_{1}\right\|_{0, \mu}$, there exists a constant $C_{14}>0$ such that

$$
\left\|\varphi_{1}\right\|_{0, \mu} \leq C_{14} L^{m-2}(m-2) ! .
$$

The proof is similar as that of (18) for $\left\|f_{1}\right\|_{0, \mu}$, so we omit the details.

Next for $\left\|\varphi_{2}\right\|_{0, \mu}$, by ([6), we write

$$
\left\|\varphi_{2}\right\|_{0, \mu} \leq 2 \sigma \sum_{1 \leq n \leq m}\left\|\partial_{q}^{m-n} h_{q q}\right\|_{0, \mu}\left\|\partial_{q}^{n}\left(h_{p}^{2}\left(1+h_{q}^{2}\right)^{-3 / 2}\right)\right\|_{0, \mu} .
$$

By the induction assumption (10), one has

$$
\forall 1 \leq n \leq m-2, \quad\left\|\partial_{q}^{m-n} h_{q q}\right\|_{0, \mu} \leq\left\|\partial_{q}^{m-n} h\right\|_{2, \mu} \leq L^{m-n-1}(m-n-2) !,
$$

and

$$
\forall 3 \leq n \leq m, \quad\left\|\partial_{q}^{n} h_{p}\right\|_{0, \mu} \leq\left\|\partial_{q}^{n-1} h\right\|_{2, \mu} \leq L^{n-2}(n-3) ! .
$$

In view of (34), we use Lemma 3.4 with $\ell=2, k_{0}=m, H=L, u_{1}=u_{2}=h_{p}$ and $u_{3}=1$, to conclude

$$
\forall 3 \leq n \leq m, \quad\left\|\partial_{q}^{n} h_{p}^{2}\right\|_{0, \mu} \leq C_{15} L^{n-2}(n-3) !
$$

To estimate the norm $\left\|\partial_{q}^{n}\left(h_{p}^{2}\left(1+h_{q}^{2}\right)^{-3 / 2}\right)\right\|_{0, \mu}$, we use the following lemma and refer to [16, Lemma 5.2] for its proof.

Lemma 3.5. Let $C_{*} \geq 1$ be the constant given in Lemma 3.4, and let $k_{0} \in \mathbb{N}$ with $k_{0} \geq 3$. Suppose $\partial_{q}^{k} u \in C^{0, \mu}(\bar{R})$ for any $k \leq k_{0}$. If there exist two constants $C_{0}$ and $\tilde{H}$ satisfying

$$
C_{0} \geq C_{*}\left(2\left\|\left(1+u^{2}\right)^{-1}\right\|_{2, \mu}+2\left\|\left(1+u^{2}\right)^{-3 / 2}\right\|_{2, \mu}+\left\|\partial_{q}\left(u^{2}\right)\right\|_{2, \mu}+1\right)^{6}
$$

and

$$
\tilde{H} \geq 2 C_{0}^{2}+\left\|\partial_{q}^{3}\left(\left(1+u^{2}\right)^{-1}\right)\right\|_{0, \mu}+\left\|\partial_{q}^{3}\left(\left(1+u^{2}\right)^{-3 / 2}\right)\right\|_{0, \mu}
$$

such that

$$
\forall 3 \leq k \leq k_{0}, \quad\left\|\partial_{q}^{k}\left(u^{2}\right)\right\|_{0, \mu} \leq C_{0} \tilde{H}^{k-2}(k-3) !
$$

then

$$
\forall 3 \leq k \leq k_{0}, \quad\left\|\partial_{q}^{k}\left(\left(1+u^{2}\right)^{-3 / 2}\right)\right\|_{0, \mu} \leq C_{0}^{2} \tilde{H}^{k-2}(k-3) !
$$


Since by the induction assumption (10), one has

$$
\forall 3 \leq n \leq m, \quad\left\|\partial_{q}^{n} h_{q}\right\|_{0, \mu} \leq\left\|\partial_{q}^{n-1} h\right\|_{2, \mu} \leq L^{n-2}(n-3) !,
$$

applying Lemma 3.4 with $\ell=2, u_{1}=u_{2}=h_{q}, k_{0}=m$ and $H=L$ gives

$$
\forall 3 \leq n \leq m, \quad\left\|\partial_{q}^{n} h_{q}^{2}\right\|_{0, \mu} \leq C_{*}\left(2\left\|h_{q}\right\|_{2, \mu}+1\right)^{2} L^{n-2}(n-3) ! \leq C_{0} L^{n-2}(n-3) !,
$$

where in the last inequality we chose

$$
C_{0} \geq C_{*}\left(2\left\|\left(1+h_{q}^{2}\right)^{-1}\right\|_{2, \mu}+2\left\|\left(1+h_{q}^{2}\right)^{-3 / 2}\right\|_{2, \mu}+\left\|\partial_{q}\left(h_{q}^{2}\right)\right\|_{2, \mu}+2\left\|h_{q}\right\|_{2, \mu}+1\right)^{6} .
$$

If we choose $L$ large enough such that

$$
L \geq 2 C_{0}^{2}+\left\|\partial_{q}^{3}\left(\left(1+h_{q}^{2}\right)^{-1}\right)\right\|_{0, \mu}+\left\|\partial_{q}^{3}\left(\left(1+h_{q}^{2}\right)^{-3 / 2}\right)\right\|_{0, \mu}
$$

then we can use the above lemma 3.5, with $u=h_{q}, k_{0}=m$ and $\tilde{H}=L$, to get

$$
\forall 3 \leq n \leq m, \quad\left\|\partial_{q}^{n}\left(\left(1+h_{q}^{2}\right)^{-3 / 2}\right)\right\|_{0, \mu} \leq C_{0}^{2} L^{n-2}(n-3) ! .
$$

Now in view of (35) and (41), applying Lemma 3.4, with $\ell=2, u_{1}=C_{15}^{-1} h_{p}^{2}$ and $u_{2}=C_{0}^{-2}(1+$ $\left.h_{q}^{2}\right)^{-3 / 2}$, yields

$$
\forall 3 \leq n \leq m, \quad\left\|\partial_{q}^{n}\left(h_{p}^{2}\left(1+h_{q}^{2}\right)^{-3 / 2}\right)\right\|_{0, \mu} \leq C_{16} L^{n-2}(n-3) ! .
$$

With (33) and (42) in hand, we can write (32) as

$$
\left\|\varphi_{2}\right\|_{0, \mu} \leq 2 \sigma\left(\sum_{1 \leq n \leq 2}+\sum_{3 \leq n \leq m-2}+\sum_{m-1 \leq n \leq m}\right)\left\|\partial_{q}^{m-n} h_{q q}\right\|_{0, \mu}\left\|\partial_{q}^{n}\left(h_{p}^{2}\left(1+h_{q}^{2}\right)^{-3 / 2}\right)\right\|_{0, \mu}
$$

and argue as in the previous steps to get

$$
\left\|\varphi_{2}\right\|_{0, \mu} \leq C_{17} L^{m-2}(m-2) !
$$

Choosing $C_{4}=C_{14}+C_{17}$, we derive the estimate (31).

Now we come back to the proof of Proposition 3.1. Choose $L$ in such a way that

$L \geq \mathcal{C}\left(C_{1}+C_{2}+C_{3}+C_{4}\right)+2 C_{0}^{2}+\left\|\partial_{q}^{3}\left(\left(1+h_{q}^{2}\right)^{-1}\right)\right\|_{0, \mu}+\left\|\partial_{q}^{3}\left(\left(1+h_{q}^{2}\right)^{-3 / 2}\right)\right\|_{0, \mu}+\left\|\partial_{q}^{2} h\right\|_{2, \mu}+1$ with $\mathcal{C}, C_{1}, \cdots, C_{4}, C_{0}$ the constants given in (16), (17), (18), (24), (31) and (40). Then combining (16), (17), (18), (24) and (31), we have,

$$
\left\|\partial_{q}^{m} h\right\|_{2, \mu} \leq \mathcal{C}\left(C_{1}+C_{2}+C_{3}+C_{4}\right) L^{m-2}(m-2) ! \leq L^{m-1}(m-2) ! .
$$

The validity of $\left(E_{m}\right)$ follows. Thus the proof of Proposition 3.1 is complete. 


\section{Gevrey regularity of the pseudo-stream function}

Let $G^{s}\left(\left[p_{0}, 0\right]\right), s \geq 1$, be the Gevrey class; see Definition 2.1 of Gevrey function. In this section we assume $\beta, \rho \in G^{s}\left(\left[p_{0}, 0\right]\right)$. Then by the alternative characterization of Gevrey function, for any $p \in\left[p_{0}, 0\right]$ we can find a neighborhood $U_{p}$ of $p$ and a constant $M_{p}$ such that

$$
\forall k \in \mathbb{N}, \quad \sup _{t \in U_{p} \cap\left[p_{0}, 0\right]}\left|\partial_{p}^{k} \beta(t)\right| \leq M_{p}^{k+1}(k !)^{s} .
$$

Note $\left[p_{0}, 0\right]$ is compact in $\mathbb{R}$; this allows us to find a constant $M$ such that

$$
\forall k \in \mathbb{N}, \quad \sup _{p \in\left[p_{0}, 0\right]}\left|\partial_{p}^{k} \beta(p)\right| \leq M^{k+1}(k !)^{s} .
$$

Similarly we can find a constant $N$ such that

$$
\forall k \in \mathbb{N}, \quad \sup _{p \in\left[p_{0}, 0\right]}\left|\partial_{p}^{k} \rho(p)\right| \leq N^{k+1}(k !)^{s} .
$$

We prove now Gevrey regularity of the pseudo-stream function, i.e., Theorem 2.3 . In view of Proposition 2.5, it suffices to show the following result for the height function $h(q, p)$.

Proposition 4.1. Let $\beta, \rho \in G^{s}\left(\left[p_{0}, 0\right]\right)$ with $s \geq 1$, and let $h \in C^{2, \mu}(\bar{R})$ be a solution to (4a)- 44 ). Then there exist two constants $L_{1}, L_{2}$ with $L_{2} \geq L_{1} \geq 1$, such that for any $m \geq 2$ we have the following estimate

$$
\left(F_{m}\right): \quad \forall \alpha=\left(\alpha_{1}, \alpha_{2}\right) \in \mathbb{N}^{2}, \quad|\alpha|=m, \quad\left\|\partial^{\alpha} h\right\|_{2} \leq L_{1}^{\alpha_{1}-1} L_{2}^{\alpha_{2}}[(|\alpha|-2) !]^{s} .
$$

Recall $\|\cdot\|_{2}$ stands for the Hölder norm $\|\cdot\|_{C^{2,0}(\bar{R})}$. Thus $h \in G^{s}(\bar{R})$; in particular if $s=1$ then $h$ is analytic in $\bar{R}$.

Remark 4.2. As to be seen in the proof, the constants $L_{1}, L_{2}$ depend on the constant $L$ given in Proposition 3.1 and the constants $M, N$ in (43) and (44), but independent of the order $m$ of derivative.

Remark 4.3. Note $\beta, \rho \in G^{s}\left(\left[p_{0}, 0\right]\right) \subset C^{\infty}\left(\left[p_{0}, 0\right]\right)$. By Remark 3.3 we see $\partial_{q} h \in C^{2, \mu}(\bar{R})$. Then differentiating the equation (4a) with respect to $p$, we can obtain $h \in C^{3, \mu}(\bar{R})$; see [4] for details. Repeating this procedure gives $h \in C^{k, \mu}(\bar{R})$ for any $k \in \mathbb{N}$, since $\beta, \rho \in C^{\infty}\left(\left[p_{0}, 0\right]\right)$.

To confirm the last statement in the above proposition 4.1, we choose $C$ in such a way that

$$
C=\max \left\{L_{1}, L_{2},\|h\|_{1, \mu}\right\},
$$

which, along with the estimate $\left(F_{m}\right)$ with $m \geq 2$ in Proposition 4.1, yields

$$
\forall \alpha \in \mathbb{N}^{2}, \quad \max _{(q, p) \in \bar{R}}\left|\partial^{\alpha} h(q, p)\right| \leq C^{|\alpha|+1}(|\alpha| !)^{s} .
$$

This gives $h \in G^{s}(\bar{R})$.

In order to prove Proposition 4.1, we need the following technical lemma and will present its proof at the end of this section. 
Lemma 4.4. Let $s \geq 1$, and $H_{1}$ and $H_{2}$ be two constants with $H_{2} \geq H_{1} \geq 1$. Suppose that $\alpha_{0}$ is a given multi-index with $\left|\alpha_{0}\right| \geq 3$, and $u, v, w \in C^{\left|\alpha_{0}\right|, \mu}(\bar{R})$. For $j=0,1,2$, denote

$\mathcal{A}_{j}=\left\{f \in C^{\left|\alpha_{0}\right|, \mu}(\bar{R})\left|\forall \alpha=\left(\alpha_{1}, \alpha_{2}\right) \leq \alpha_{0}, \quad\right| \alpha \mid \geq j+1, \quad\left\|\partial^{\alpha} f\right\|_{0} \leq H_{1}^{\alpha_{1}-j} H_{2}^{\alpha_{2}}[(|\alpha|-j-1) !]^{s}\right\}$.

Then there exists a constant $c_{*}$, depending only on the $C^{2,0}$-norms of $u, v$ and $w$, but independent of $\alpha_{0}$, such that

(a) if $u \in \mathcal{A}_{2}$ and $v \in \mathcal{A}_{1}$, then $c_{*}^{-1} u v \in \mathcal{A}_{1}$, that is,

$$
\forall \alpha=\left(\alpha_{1}, \alpha_{2}\right) \leq \alpha_{0}, \quad|\alpha| \geq 2, \quad\left\|\partial^{\alpha}(u v)\right\|_{0} \leq c_{*} H_{1}^{\alpha_{1}-1} H_{2}^{\alpha_{2}}[(|\alpha|-2) !]^{s} ;
$$

if additionally $w \in \mathcal{A}_{2}$ then $c_{*}^{-1} u v w \in \mathcal{A}_{1}$;

(b) if $u \in \mathcal{A}_{2}$ and $v, w \in \mathcal{A}_{1}$, then $c_{*}^{-1} u v w \in \mathcal{A}_{0}$;

(c) if $u, v \in \mathcal{A}_{2}$ and $w \in \mathcal{A}_{0}$, then $c_{*}^{-1} u v w \in \mathcal{A}_{0}$;

(d) if $u, v, w \in \mathcal{A}_{2}$, then $c_{*}^{-1} H_{1} u v w \in \mathcal{A}_{1}$, that is,

$$
\forall \alpha=\left(\alpha_{1}, \alpha_{2}\right) \leq \alpha_{0}, \quad|\alpha| \geq 2, \quad\left\|\partial^{\alpha}(u v w)\right\|_{0} \leq c_{*} H_{1}^{\alpha_{1}-2} H_{2}^{\alpha_{2}}[(|\alpha|-2) !]^{s} ;
$$

(e) if $u \in \mathcal{A}_{3}$ and $v \in \mathcal{A}_{2}$, then $\tilde{c}_{*}^{-1} u v \in \mathcal{A}_{2}$, with $\tilde{c}_{*}$ a constant depending on the $C^{3,0}$-norm of $u$ and $C^{2,0}$-norm of $v$.

Now we prove Proposition 4.1 .

Proof of Proposition 4.1. In view of Remark 4.3 we may assume that $h \in C^{k, \mu}(\bar{R})$ for any $k \in \mathbb{N}$. We now use induction on $m$ to prove the estimate $\left(F_{m}\right)$. First for $m=2,\left(F_{m}\right)$ obviously holds by choosing $L_{1}, L_{2}$ in such a way that

$$
L_{2} \geq L_{1} \geq\|h\|_{4}+1 .
$$

Next let $m \geq 3$ and assume that $\left(F_{j}\right)$ holds for any $j$ with $2 \leq j \leq m-1$, that is,

$$
\forall \gamma=\left(\gamma_{1}, \gamma_{2}\right), 2 \leq \gamma_{1}+\gamma_{2} \leq m-1, \quad\left\|\partial^{\gamma} h\right\|_{2} \leq L_{1}^{\gamma_{1}-1} L_{1}^{\gamma_{2}}[(|\gamma|-2) !]^{s} .
$$

We have to prove the validity of $\left(F_{m}\right)$. This is equivalent to show the following estimate

$$
\left(F_{m, n}\right): \quad\left\|\partial_{q}^{m-n} \partial_{p}^{n} h\right\|_{2} \leq L_{1}^{m-n-1} L_{2}^{n}[(m-2) !]^{s}
$$

holds for all $n$ with $0 \leq n \leq m$.

In what follows we use induction on $n$ to show (47) with fixed $m \geq 3$. Firstly note that $s \geq 1$, and thus from Proposition 3.1 we see that $\left(F_{m, 0}\right)$ holds if we choose

$$
L_{1} \geq L
$$

Next let $1 \leq n \leq m$ and assume that $\left(F_{m, i}\right)$ holds for all $i$ with $0 \leq i \leq n-1$, that is,

$$
\forall 0 \leq i \leq n-1, \quad\left\|\partial_{q}^{m-i} \partial_{p}^{i} h\right\|_{2} \leq L_{1}^{m-i-1} L_{2}^{i}[(m-2) !]^{s} .
$$


We have to show $\left(F_{m, n}\right)$ holds as well, i.e., to prove that

$$
\left\|\partial_{q}^{m-n} \partial_{p}^{n} h\right\|_{2} \leq L_{1}^{m-n-1} L_{2}^{n}[(m-2) !]^{s} .
$$

To do so, we firstly compute, with $1 \leq n \leq m$,

$$
\begin{aligned}
\left\|\partial_{q}^{m-n} \partial_{p}^{n} h\right\|_{2} & \leq\left\|\partial_{q}^{m-n} \partial_{p}^{n} h\right\|_{1}+\left\|\partial_{q}^{m-n+2} \partial_{p}^{n} h\right\|_{0}+\left\|\partial_{q}^{m-n+1} \partial_{p}^{n+1} h\right\|_{0}+\left\|\partial_{q}^{m-n} \partial_{p}^{n+2} h\right\|_{0} \\
& \leq\left\|\partial_{q}^{m-n} \partial_{p}^{n-1} h\right\|_{2}+2\left\|\partial_{q}^{m-(n-1)} \partial_{p}^{n-1} h\right\|_{2}+\left\|\partial_{q}^{m-n} \partial_{p}^{n+2} h\right\|_{0} .
\end{aligned}
$$

The induction assumptions (46) and (49) yield

$$
\begin{aligned}
\left\|\partial_{q}^{m-n} \partial_{p}^{n-1} h\right\|_{2}+2\left\|\partial_{q}^{m-(n-1)} \partial_{p}^{n-1} h\right\|_{2} & \leq L_{1}^{m-n-1} L_{2}^{n-1}[(m-3) !]^{s}+2 L_{1}^{m-n} L_{2}^{n-1}[(m-2) !]^{s} \\
& \leq L_{2}^{-1}\left(1+2 L_{1}\right) L_{1}^{m-n-1} L_{2}^{n}[(m-2) !]^{s} \\
& \leq \frac{1}{2} L_{1}^{m-n-1} L_{2}^{n}[(m-2) !]^{s}
\end{aligned}
$$

where in the last inequality we choose

$$
L_{2} \geq 8 L_{1} \geq 8
$$

Accordingly, in order to obtain (50), it suffices to prove

$$
\left\|\partial_{q}^{m-n} \partial_{p}^{n+2} h\right\|_{0} \leq \frac{1}{2} L_{1}^{m-n-1} L_{2}^{n}[(m-2) !]^{s} .
$$

The rest is occupied by the proof of the above estimate.

From now on we fix $m$ and $n$ with $m \geq 3$ and $1 \leq n \leq m$, and denote $\alpha=\left(\alpha_{1}, \alpha_{2}\right)=$ $(m-n, n)$. Applying $\partial^{\alpha}=\partial_{q}^{m-n} \partial_{p}^{n}$ on both sides of the equation (4a) gives

$$
\begin{aligned}
\left(1+h_{q}^{2}\right)\left(\partial^{\alpha} h_{p p}\right)= & -\sum_{\gamma \leq \alpha, \gamma \neq 0}\left(\begin{array}{l}
\alpha \\
\gamma
\end{array}\right)\left(\partial^{\gamma} h_{q}^{2}\right)\left(\partial^{\alpha-\gamma} h_{p p}\right)+2 \partial^{\alpha}\left(h_{p} h_{q} h_{q p}\right)-\partial^{\alpha}\left(h_{p}^{2} h_{q q}\right) \\
& +g \partial^{\alpha}\left((h-d) \rho_{p} h_{p}^{3}\right)-\partial^{\alpha}\left(\beta h_{p}^{3}\right)
\end{aligned}
$$

which implies

$$
\begin{aligned}
& \left\|\left(1+h_{q}^{2}\right)\left(\partial^{\alpha} h_{p p}\right)\right\|_{0} \leq \sum_{\gamma \leq \alpha, \gamma \neq 0}\left(\begin{array}{l}
\alpha \\
\gamma
\end{array}\right)\left\|\partial^{\gamma} h_{q}^{2}\right\|_{0}\left\|\partial^{\alpha-\gamma} h_{p p}\right\|_{0}+2\left\|\partial^{\alpha}\left(h_{p} h_{q} h_{q p}\right)\right\|_{0} \\
& +\left\|\partial^{\alpha}\left(h_{p}^{2} h_{q q}\right)\right\|_{0}+g\left\|(h-d) \rho_{p} h_{p}^{3}\right\|_{0}+\left\|\partial^{\alpha}\left(\beta h_{p}^{3}\right)\right\|_{0} .
\end{aligned}
$$

Since

$$
\left\|\partial_{q}^{m-n} \partial_{p}^{n+2} h\right\|_{0}=\left\|\partial^{\alpha} h_{p p}\right\|_{0} \leq\left\|\left(1+h_{q}^{2}\right)\left(\partial^{\alpha} h_{p p}\right)\right\|_{0},
$$

we obtain, with $\alpha=\left(\alpha_{1}, \alpha_{2}\right)=(m-n, n)$,

$$
\begin{aligned}
\left\|\partial_{q}^{m-n} \partial_{p}^{n+2} h\right\|_{0} \leq & \sum_{\gamma \leq \alpha, \gamma \neq 0}\left(\begin{array}{l}
\alpha \\
\gamma
\end{array}\right)\left\|\partial^{\gamma} h_{q}^{2}\right\|_{0}\left\|\partial^{\alpha-\gamma} h_{p p}\right\|_{0}+2\left\|\partial^{\alpha}\left(h_{p} h_{q} h_{q p}\right)\right\|_{0} \\
& +\left\|\partial^{\alpha}\left(h_{p}^{2} h_{q q}\right)\right\|_{0}+g\left\|(h-d) \rho_{p} h_{p}^{3}\right\|_{0}+\left\|\partial^{\alpha}\left(\beta h_{p}^{3}\right)\right\|_{0} .
\end{aligned}
$$


We now treat the terms on the right-hand side through the following lemmas.

To simplify the notations, we will use $c_{j}, j \geq 1$, to denote suitable harmless constants larger than 1. By harmless constants it means that these constants are independent of $m$ and $n$. The following three lemmas, Lemma 4.5-Lemma 4.7, have been proved in [16]. For completeness, we present their proofs here again.

Lemma 4.5. For $\alpha=\left(\alpha_{1}, \alpha_{2}\right)=(m-n, n)$ with $1 \leq n \leq m$, we have

$$
\sum_{\gamma \leq \alpha, \gamma \neq 0}\left(\begin{array}{c}
\alpha \\
\gamma
\end{array}\right)\left\|\partial^{\gamma} h_{q}^{2}\right\|_{0}\left\|\partial^{\alpha-\gamma} h_{p p}\right\|_{0} \leq c_{1} L_{1}^{\alpha_{1}-2} L_{2}^{\alpha_{2}}[(|\alpha|-2) !]^{s} .
$$

Proof of the lemma. We firstly use Lemma 4.4 to treat the term $\left\|\partial^{\gamma} h_{q}^{2}\right\|_{0}$ with $3 \leq|\gamma| \leq|\alpha|=m$. To do so, write $\gamma=\tilde{\gamma}+(\gamma-\tilde{\gamma})$ with $|\tilde{\gamma}|=|\gamma|-1 \geq 2$. Without loss of generality we may take $\gamma-\tilde{\gamma}=(0,1)$, and the arguments below also holds when $\gamma-\tilde{\gamma}=(1,0)$. Thus

$$
\partial^{\gamma} h_{q}^{2}=2 \partial^{\tilde{\gamma}}\left(h_{q} h_{q p}\right) .
$$

Note that for any $\xi=\left(\xi_{1}, \xi_{2}\right) \leq \tilde{\gamma}$ with $|\xi| \geq 3$, we have, using the induction assumption (46),

$$
\left\|\partial^{\xi} h_{q p}\right\|_{0} \leq\left\|\partial^{\xi} h\right\|_{2} \leq L_{1}^{\xi_{1}-1} L_{2}^{\xi_{2}}[(|\xi|-2) !]^{s},
$$

and

$$
\left\|\partial^{\xi} h_{q}\right\|_{0} \leq \begin{cases}\left\|\partial_{q}^{\xi_{1}} \partial_{p}^{\xi_{2}-1} h\right\|_{2} \leq L_{1}^{\xi_{1}-1} L_{2}^{\xi_{2}-1}[(|\xi|-3) !]^{s} \leq L_{1}^{\xi_{1}-2} L_{2}^{\xi_{2}}[(|\xi|-3) !]^{s}, & \xi_{2} \geq 1, \\ \left\|\partial_{q}^{\xi_{1}-1} h\right\|_{2} \leq L_{1}^{\xi_{1}-2}[(|\xi|-3) !]^{s}=L_{1}^{\xi_{1}-2} L_{2}^{\xi_{2}}[(|\xi|-3) !]^{s}, & \xi_{2}=0,\end{cases}
$$

where in the case $\xi_{2} \geq 1$ we used $L_{2} \geq L_{1}$. Therefore applying Lemma 4.4-(aa), with $H_{1}=L_{1}$, $H_{2}=L_{2}, u=h_{q}$ and $v=h_{q p}$, gives

$$
\left\|\partial^{\tilde{\gamma}}\left(h_{q} h_{q p}\right)\right\|_{0} \leq c_{5} L_{1}^{\tilde{\gamma}_{1}-1} L_{2}^{\tilde{\gamma}_{2}}[(|\tilde{\gamma}|-2) !]^{s}=c_{5} L_{1}^{\gamma_{1}-1} L_{2}^{\gamma_{2}-1}[(|\gamma|-3) !]^{s} \leq c_{5} L_{1}^{\gamma_{1}-2} L_{2}^{\gamma_{2}}[(|\gamma|-3) !]^{s},
$$

the last inequality holding because $L_{2} \geq L_{1}$. This along with the relation (55) yields

$$
\forall \gamma, 3 \leq|\gamma| \leq|\alpha|, \quad\left\|\partial^{\gamma} h_{q}^{2}\right\|_{0} \leq 2 c_{5} L_{1}^{\gamma_{1}-2} L_{2}^{\gamma_{2}}[(|\gamma|-3) !]^{s} .
$$

On the other hand, for the term $\left\|\partial^{\alpha-\gamma} h_{p p}\right\|_{0}$, we have, by the induction assumption (46),

$$
\forall \gamma \leq \alpha, 1 \leq|\gamma| \leq|\alpha|-2, \quad\left\|\partial^{\alpha-\gamma} h_{p p}\right\|_{0} \leq\left\|\partial^{\alpha-\gamma} h\right\|_{2} \leq L_{1}^{\alpha_{1}-\gamma_{1}-1} L_{2}^{\alpha_{2}-\gamma_{2}}[(|\alpha|-|\gamma|-2) !]^{s} .
$$

Next we write

$$
\begin{aligned}
\sum_{\gamma \leq \alpha, \gamma \neq 0}\left(\begin{array}{l}
\alpha \\
\gamma
\end{array}\right)\left\|\partial^{\gamma} h_{q}^{2}\right\|_{0}\left\|\partial^{\alpha-\gamma} h_{p p}\right\|_{0} & =\left(\sum_{\substack{\gamma \leq \alpha \\
1 \leq|\gamma| \leq 2}}+\sum_{\substack{\gamma \leq|\gamma| \leq|\alpha|-2 \\
\gamma \leq \alpha}}+\sum_{\substack{\gamma \leq \alpha \\
|\gamma| \leq|\alpha|-1}}\right)\left(\begin{array}{l}
\alpha \\
\gamma
\end{array}\right)\left\|\partial^{\gamma} h_{q}^{2}\right\|_{0}\left\|\partial^{\alpha-\gamma} h_{p p}\right\|_{0} \\
& =J_{1}+J_{2}+J_{3} .
\end{aligned}
$$

By virtue of (57) and (58), direct computation as in (23), shows that

$$
J_{1}+J_{3} \leq c_{6} L_{1}^{\alpha_{1}-2} L_{2}^{\alpha_{2}}[(|\alpha|-2) !]^{s} .
$$


Next for $J_{2}$, which appears only when $|\alpha| \geq 5$, we have by (57) and (58) that

$$
\begin{aligned}
J_{2} & \leq 2 c_{5} \sum_{\substack{\gamma \leq \alpha \\
3 \leq|\gamma| \leq|\alpha|-2}} \frac{|\alpha| !}{|\gamma| !(|\alpha|-|\gamma|) !} L_{1}^{\gamma_{1}-2} L_{2}^{\gamma_{2}}[(|\gamma|-3) !]^{s} L_{1}^{\alpha_{1}-\gamma_{1}-1} L_{2}^{\alpha_{2}-\gamma_{2}}[(|\alpha|-|\gamma|-2) !]^{s} \\
& \leq c_{7} \sum_{\substack{\gamma \leq \alpha \\
3 \leq|\gamma| \leq|\alpha|-2}} \frac{|\alpha| !}{|\gamma|^{3}(|\alpha|-|\gamma|)^{2}} L_{1}^{\alpha_{1}-3} L_{2}^{\alpha_{2}}[(|\gamma|-3) !]^{s-1}[(|\alpha|-|\gamma|-2) !]^{s-1} \\
& \leq c_{7} L_{1}^{\alpha_{1}-3} L_{2}^{\alpha_{2}} \sum_{\substack{\gamma \leq \alpha \\
3 \leq|\gamma| \leq|\alpha|-2}} \frac{|\alpha| !}{|\gamma|^{3}(|\alpha|-|\gamma|)^{2}}[(|\alpha|-5) !]^{s-1} \\
& \leq c_{7} L_{1}^{\alpha_{1}-3} L_{2}^{\alpha_{2}}[(|\alpha|-2) !]^{s} \sum_{\substack{\gamma \leq \alpha \\
3 \leq|\gamma| \leq|\alpha|-2}} \frac{|\gamma|^{3}(|\alpha|-|\gamma|)^{2}}{\mid{ }^{2}} \\
& \leq c_{8} L_{1}^{\alpha_{1}-3} L_{2}^{\alpha_{2}}[(|\alpha|-2) !]^{s}, \quad
\end{aligned}
$$

the last inequality using Lemma 2.6. Therefore, choosing $c_{1}=c_{6}+c_{8}$, we can combine the estimates for $J_{1}, J_{2}$ and $J_{3}$ to complete the proof of the lemma.

Lemma 4.6. For $\alpha=\left(\alpha_{1}, \alpha_{2}\right)=(m-n, n)$ with $1 \leq n \leq m$, we have

$$
2\left\|\partial^{\alpha}\left(h_{p} h_{q} h_{q p}\right)\right\|_{0}+\left\|\partial^{\alpha}\left(h_{p}^{2} h_{q q}\right)\right\|_{0} \leq c_{2} L_{1}^{\alpha_{1}} L_{2}^{\alpha_{2}-1}[(|\alpha|-2) !]^{s} .
$$

Proof of the lemma. Since $n \geq 1$, we can write $\alpha=\tilde{\alpha}+(0,1)$ with $\tilde{\alpha}=\left(\tilde{\alpha}_{1}, \tilde{\alpha}_{2}\right)=(m-n, n-1)$. Thus

$$
\partial^{\alpha}\left(h_{p} h_{q} h_{q p}\right)=\partial^{\tilde{\alpha}}\left(h_{p p} h_{q} h_{q p}+h_{p} h_{q p} h_{q p}+h_{p} h_{q} h_{q p p}\right) .
$$

We next compute the estimate for the term $\partial^{\tilde{\alpha}}\left(h_{p p} h_{q} h_{q p}\right)$. For any $\gamma \leq \tilde{\alpha}$ with $|\gamma| \geq 3$, we have, as for $\left\|\partial^{\xi} h_{q}\right\|_{0}$ in (56),

$$
\left\|\partial^{\gamma} h_{q}\right\|_{0} \leq L_{1}^{\gamma_{1}-2} L_{2}^{\gamma_{2}}[(|\gamma|-3) !]^{s}, \quad\left\|\partial^{\gamma} h_{p}\right\|_{0} \leq L_{1}^{\gamma_{1}-2} L_{2}^{\gamma_{2}}[(|\gamma|-3) !]^{s},
$$

and by the induction assumption (46) and (49), in view of $\gamma_{2} \leq \tilde{\alpha}_{2}=n-1$,

$$
\begin{aligned}
\left\|\partial^{\gamma} h_{p p}\right\|_{0} & \leq\left\|\partial^{\gamma} h\right\|_{2} \leq L_{1}^{\gamma_{1}-1} L_{2}^{\gamma_{2}}[(|\gamma|-2) !]^{s} \\
\left\|\partial^{\gamma} h_{q p}\right\|_{0} & \leq\left\|\partial^{\gamma} h\right\|_{2} \leq L_{1}^{\gamma_{1}-1} L_{2}^{\gamma_{2}}[(|\gamma|-2) !]^{s} \\
\left\|\partial^{\gamma} h_{q p p}\right\|_{0} & \leq\left\|\partial_{q}^{\gamma_{1}+1} \partial_{p}^{\gamma_{2}} h\right\|_{2} \leq L_{1}^{\gamma_{1}} L_{2}^{\gamma_{2}}[(|\gamma|-1) !]^{s} .
\end{aligned}
$$

Thus we obtain, using Lemma 4.4 (国) with $u=h_{p}, w=h_{p}$ and $v=h_{q p}$, $\left\|\partial^{\tilde{\alpha}}\left(h_{p} h_{q} h_{q p}\right)\right\|_{0} \leq c_{9} L_{1}^{\tilde{\alpha}_{1}-1} L_{2}^{\tilde{\alpha}_{2}}[(|\tilde{\alpha}|-2) !]^{s}=c_{9} L_{1}^{\alpha_{1}-1} L_{2}^{\alpha_{2}-1}[(|\alpha|-3) !]^{s} \leq c_{9} L_{1}^{\alpha_{1}} L_{2}^{\alpha_{2}-1}[(|\alpha|-2) !]^{s}$.

Similarly, using Lemma 4.4-(b) with $u=h_{p}, v=w=h_{q p}$, gives

$$
\left\|\partial^{\tilde{\alpha}}\left(h_{p} h_{q p} h_{q p}\right)\right\|_{0} \leq c_{10} L_{1}^{\tilde{\alpha}_{1}} L_{2}^{\tilde{\alpha}_{2}}[(|\tilde{\alpha}|-1) !]^{s}=c_{10} L_{1}^{\alpha_{1}} L_{2}^{\alpha_{2}-1}[(|\alpha|-2) !]^{s},
$$

while using Lemma 4.4.(ㄷ) with $u=h_{p}, v=h_{q}$ and $w=h_{q p p}$ gives

$$
\left\|\partial^{\tilde{\alpha}}\left(h_{p} h_{p} h_{q p p}\right)\right\|_{0} \leq c_{11} L_{1}^{\tilde{\alpha}_{1}} L_{2}^{\tilde{\alpha}_{2}}[(|\tilde{\alpha}|-1) !]^{s}=c_{11} L_{1}^{\alpha_{1}} L_{2}^{\alpha_{2}-1}[(|\alpha|-2) !]^{s} .
$$


Combining the above inequalities, we have, in view of (59),

$$
2\left\|\partial^{\alpha}\left(h_{p} h_{q} h_{q p}\right)\right\|_{0} \leq 2\left(c_{9}+c_{10}+c_{11}\right) L_{1}^{\alpha_{1}} L_{2}^{\alpha_{2}-1}[(|\alpha|-2) !]^{s} .
$$

The treatment for the term $\left\|\partial^{\alpha}\left(h_{p}^{2} h_{q q}\right)\right\|_{0}$ is completely the same as above, so we have

$$
\left\|\partial^{\alpha}\left(h_{p}^{2} h_{q q}\right)\right\|_{0} \leq c_{12} L_{1}^{\alpha_{1}} L_{2}^{\alpha_{2}-1}[(|\alpha|-2) !]^{s} .
$$

Combining the above two estimates, we choose $c_{2}=2\left(c_{9}+c_{10}+c_{11}\right)+c_{12}$ to complete the proof of the lemma.

Lemma 4.7. Let $\beta \in G^{s}\left(\left[p_{0}, 0\right]\right)$ and $L_{2} \geq L_{1} \tilde{M}$ with $\tilde{M}$ a constant depending on $M$ given in (43) and $s$. We have, for $\alpha=\left(\alpha_{1}, \alpha_{2}\right)=(m-n, n)$ with $1 \leq n \leq m$,

$$
\left\|\partial^{\alpha}\left(\beta h_{p}^{3}\right)\right\|_{0} \leq c_{3} L_{1}^{\alpha_{1}-2} L_{2}^{\alpha_{2}}[(|\alpha|-2) !]^{s} .
$$

Proof of the lemma. As for $\left\|\partial^{\xi} h_{q}\right\|_{0}$ in (56) , we have by induction

$$
\forall \gamma \leq \alpha, \quad|\gamma| \geq 3, \quad\left\|\partial^{\gamma} h_{p}\right\|_{0} \leq L_{1}^{\gamma_{1}-2} L_{2}^{\gamma_{2}}[(|\gamma|-3) !]^{s} .
$$

Thus using Lemma 4.4.(d) with $H_{i}=L_{i}, i=1,2, u=v=w=h_{p}$, we deduce that $c_{13}^{-1} L_{1} h_{p}^{3} \in \mathcal{A}_{1}$, that is,

$$
\forall \gamma \leq \alpha, \quad|\gamma| \geq 2, \quad\left\|\partial^{\gamma}\left(h_{p}^{3}\right)\right\|_{0} \leq c_{13} L_{1}^{\gamma_{1}-2} L_{2}^{\gamma_{2}}[(|\gamma|-2) !]^{s} .
$$

On the other hand, since $\beta(p) \in G^{s}\left(\left[p_{0}, 0\right]\right)$, then using (43) gives

$$
\forall|\gamma| \geq 3, \quad\left\|\partial^{\gamma} \beta\right\|_{0} \leq \begin{cases}0, & \gamma_{1} \geq 1 \\ \left\|\partial_{p}^{\gamma_{2}} \beta\right\|_{0} \leq M^{\gamma_{2}+1}\left(\gamma_{2} !\right)^{s} \leq \tilde{M}^{\gamma_{2}}\left[\left(\gamma_{2}-3\right) !\right]^{s}, & \gamma_{1}=0\end{cases}
$$

where $\tilde{M}$ in the last inequality is a constant depending only on $M$ and $s$. Thus if we choose $L_{1}, L_{2}$ in such a way that

$$
L_{2} \geq L_{1} \tilde{M}
$$

then we have

$$
\forall \gamma \leq \alpha, \quad|\gamma| \geq 3, \quad\left\|\partial^{\gamma} \beta\right\|_{0} \leq L_{1}^{\gamma_{1}-2} L_{2}^{\gamma_{2}}[(|\gamma|-3) !]^{s} .
$$

Now we write

$$
\left\|\partial^{\alpha}\left(\beta h_{p}^{3}\right)\right\|_{0} \leq \sum_{\gamma \leq \alpha} \frac{|\alpha| !}{|\gamma| !(|\alpha|-|\gamma|) !}\left\|\partial^{\gamma} \beta\right\|_{0}\left\|\partial^{\alpha-\gamma}\left(h_{p}^{3}\right)\right\|_{0}
$$

This together with (60) and (62) allows us to argue as the treatment of $J_{1}-J_{3}$ in Lemma 4.5, to conclude

$$
\left\|\partial^{\alpha}\left(\beta h_{p}^{3}\right)\right\|_{0} \leq c_{14} L_{1}^{\alpha_{1}-2} L_{2}^{\alpha_{2}}[(|\alpha|-2) !]^{s} .
$$

Thus the desired estimate follows if choosing $c_{3}=c_{14}$. The proof is thus complete.

Lemma 4.8. Let $\rho \in G^{s}\left(\left[p_{0}, 0\right]\right)$. We have, for $\alpha=\left(\alpha_{1}, \alpha_{2}\right)=(m-n, n)$ with $1 \leq n \leq m$,

$$
g\left\|\partial^{\alpha}\left((h-d) \rho_{p} h_{p}^{3}\right)\right\|_{0} \leq c_{4} L_{1}^{\alpha_{1}-2} L_{2}^{\alpha_{2}}[(|\alpha|-2) !]^{s} .
$$


Proof of the lemma. As in the previous lemma, we have

$$
\forall \gamma \leq \alpha, \quad|\gamma| \geq 2, \quad\left\|\partial^{\gamma}\left(h_{p}^{3}\right)\right\|_{0} \leq c_{13} L_{1}^{\gamma_{1}-2} L_{2}^{\gamma_{2}}[(|\gamma|-2) !]^{s} .
$$

Next as the treatment for $\left\|\partial^{\xi} h_{q}\right\|_{0}$ in (56), we have

$$
\forall \gamma \leq \alpha, \quad|\gamma| \geq 4, \quad\left\|\partial^{\gamma}(h-d)\right\|_{0}=\left\|\partial^{\gamma} h\right\|_{0} \leq L_{1}^{\gamma_{1}-3} L_{2}^{\gamma_{2}}[(|\gamma|-4) !]^{s} .
$$

Moreover, since $\rho(p) \in G^{s}\left(\left[p_{0}, 0\right]\right)$, then using (44) gives

$$
\forall|\gamma| \geq 3, \quad\left\|\partial^{\gamma} \rho_{p}\right\|_{0} \leq \begin{cases}0, & \gamma_{1} \geq 1 \\ \left\|\partial_{p}^{\gamma_{2}+1} \rho\right\|_{0} \leq N^{\gamma_{2}+2}\left[\left(\gamma_{2}+1\right) !\right]^{s} \leq \tilde{N}^{\gamma_{2}}\left[\left(\gamma_{2}-3\right) !\right]^{s}, & \gamma_{1}=0\end{cases}
$$

with $\tilde{N}$ a constant depending only on $N$ and $s$. Thus if we choose $L_{1}, L_{2}$ in such a way that

$$
L_{2} \geq L_{1} \tilde{N}
$$

then we have

$$
\forall \gamma \leq \alpha, \quad|\gamma| \geq 3, \quad\left\|\partial^{\gamma} \rho_{p}\right\|_{0} \leq L_{1}^{\gamma_{1}-2} L_{2}^{\gamma_{2}}[(|\gamma|-3) !]^{s} .
$$

Combining (64) and (66), we can apply Lemma 4.4.(或), with $u=h-d$ and $v=\rho_{p}$, to conclude,

$$
\forall \gamma \leq \alpha, \quad|\gamma| \geq 3, \quad\left\|\partial^{\gamma}\left((h-d) \rho_{p}\right)\right\|_{0} \leq c_{15} L_{1}^{\gamma_{1}-2} L_{2}^{\gamma_{2}}[(|\gamma|-3) !]^{s} .
$$

Now we write

$$
\begin{aligned}
& g\left\|\partial^{\alpha}\left((h-d) \rho_{p} h_{p}^{3}\right)\right\|_{0} \leq g \sum_{|\gamma| \leq|\alpha|} \frac{|\alpha| !}{|\gamma| !(|\alpha|-|\gamma|) !}\left\|\partial^{\gamma}(h-d) \rho_{p}\right\|_{0}\left\|\partial^{\alpha-\gamma} h_{p}^{3}\right\|_{0} \\
& \leq g\left(\sum_{\substack{\gamma \leq \alpha \\
0 \leq|\gamma| \leq 2}}+\sum_{\substack{\gamma \leq \alpha \\
3 \leq|\gamma| \leq|\alpha|-2}}+\sum_{\substack{\gamma \leq \alpha \\
|\alpha|-1 \leq|\gamma|}}\right) \frac{|\alpha| !}{|\gamma| !(|\alpha|-|\gamma|) !}\left\|\partial^{\gamma}(h-d) \rho_{p}\right\|_{0}\left\|\partial^{\alpha-\gamma} h_{p}^{3}\right\|_{0} .
\end{aligned}
$$

Again arguing as the treatment of $J_{1}-J_{3}$ in Lemma 4.5, we use (63) and (67) to conclude

$$
g\left\|\partial^{\alpha}\left((h-d) \rho_{p} h_{p}^{3}\right)\right\|_{0} \leq c_{4} L_{1}^{\alpha_{1}-2} L_{2}^{\alpha_{2}}[(|\alpha|-2) !]^{s},
$$

completing the proof of the lemma.

We now continue the proof of Proposition 4.1, Combining (154) and the conclusions in the previous four lemmas, Lemma 4.5-Lemma 4.8, we get

$$
\begin{aligned}
\left\|\partial_{q}^{m-n} \partial_{p}^{n+2} h\right\|_{0} & \leq\left(\left(c_{1}+c_{3}+c_{4}\right) L_{1}^{-1}+c_{2} L_{1} L_{2}^{-1}\right) L_{1}^{\alpha_{1}-1} L_{2}^{\alpha_{2}}[(|\alpha|-2) !]^{s} \\
& \leq \frac{1}{2} L_{1}^{\alpha_{1}-1} L_{2}^{\alpha_{2}}[(|\alpha|-2) !]^{s},
\end{aligned}
$$

where in the last inequality we chose

$$
L_{1} \geq 4\left(c_{1}+c_{3}+c_{4}\right), \quad L_{2} \geq 4 c_{2} L_{1} .
$$


Then we get the desired estimate (52), and thus the validity of $\left(F_{m, n}\right)$ and $\left(F_{m}\right)$. Summarizing the relations (45), (48), (51), (61), (65) and (68), we can choose

$$
L_{1} \geq \max \left\{L,\|h\|_{4}+1,4\left(c_{1}+c_{3}+c_{4}\right)\right\} \text { and } L_{2} \geq\left(8+4 c_{2}+\tilde{M}+\tilde{N}\right) L_{1},
$$

with $\tilde{M}, \tilde{N}$ the constants appearing respectively in (61) and (65), to complete the proof of Proposition 4.1 .

The rest of this section is devoted to

Proof of Lemma 4.4. We refer to [16, Lemma 4.4] for the proof of the conclusions (国)-(的). Now we only prove the final statement (国). To simplify the notations, we use $\|\cdot\|$ in this proof to stand for the norm $\|\cdot\|_{C^{0}(\bar{R})}$, and use $a_{j}, j \geq 1$, to denote different suitable harmless constants larger than 1 , which depend on the $C^{3,0}$-norm of $u$ and $C^{2,0}$-norm of $v$, but are independent of the order $\alpha_{0}$ of derivative.

Assume $u \in \mathcal{A}_{3}$ and $v \in \mathcal{A}_{2}$. By Leibniz formula we have, for any $\alpha \leq \alpha_{0}$ with $|\alpha| \geq 3$,

$$
\partial^{\alpha}(u v)=\sum_{0 \leq \beta \leq \alpha}\left(\begin{array}{l}
\alpha \\
\beta
\end{array}\right)\left(\partial^{\beta} u\right)\left(\partial^{\alpha-\beta} v\right)
$$

Then

$$
\left\|\partial^{\alpha}(u v)\right\| \leq \sum_{0 \leq \beta \leq \alpha} \frac{|\alpha| !}{|\beta| !|\alpha-\beta| !}\left\|\partial^{\beta} u\right\|\left\|\partial^{\alpha-\beta} v\right\|=I_{1}+I_{2}+I_{3},
$$

with

$$
\begin{aligned}
& I_{1}=\sum_{\substack{0 \leq \beta \leq \alpha \\
|\beta| \leq 3}} \frac{|\alpha| !}{|\beta| !|\alpha-\beta| !}\left\|\partial^{\beta} u\right\|\left\|\partial^{\alpha-\beta} v\right\|, \\
& I_{2}=\sum_{\substack{0 \leq \beta \leq \alpha \\
4 \leq|\beta| \leq|\alpha|-3}} \frac{|\alpha| !}{|\beta| !|\alpha-\beta| !}\left\|\partial^{\beta} u\right\|\left\|\partial^{\alpha-\beta} v\right\|, \\
& I_{3}=\sum_{\substack{0 \leq \beta \leq \alpha \\
|\beta| \geq|\alpha|-2}} \frac{|\alpha| !}{|\beta| !|\alpha-\beta| !}\left\|\partial^{\beta} u\right\|\left\|\partial^{\alpha-\beta} v\right\| .
\end{aligned}
$$

Since $H_{2} \geq H_{1}$, direct computation shows that there exists $a_{1}>1$ such that

$$
I_{1}+I_{3} \leq a_{1}\left(\|u\|_{3}+\|v\|_{2}+1\right)^{2} H_{1}^{\alpha_{1}-2} H_{2}^{\alpha_{2}}[(|\alpha|-3) !]^{s} .
$$


For $I_{2}$, which appears only when $|\alpha| \geq 7$, we have

$$
\begin{aligned}
I_{2} & \leq \sum_{\substack{0 \leq \beta \leq \alpha \\
4 \leq|\beta| \leq|\alpha|-3}} \frac{|\alpha| !}{|\beta| !|\alpha-\beta| !} H_{1}^{\beta_{1}-3} H_{2}^{\beta_{2}}((|\beta|-4) !)^{s} H_{1}^{\alpha_{1}-\beta_{1}-2} H_{2}^{\alpha_{2}-\beta_{2}}[(|\alpha|-|\beta|-3) !]^{s} \\
& \leq a_{2} \sum_{\substack{0 \leq \beta \leq \alpha \\
4 \leq|\beta| \leq|\alpha|-3}} \frac{|\alpha| !}{|\beta|^{4}|\alpha-\beta|^{3}} H_{1}^{\alpha_{1}-5} H_{2}^{\alpha_{2}}[(|\beta|-4) !]^{s-1}[(|\alpha|-|\beta|-3) !]^{s-1} \\
& \leq a_{2} H_{1}^{\alpha_{1}-5} H_{2}^{\alpha_{2}} \sum_{\substack{0 \leq \beta \leq \alpha \\
4 \leq|\beta| \leq|\alpha|-3}} \frac{|\alpha| !}{|\beta|^{4}|\alpha-\beta|^{3}}[(|\alpha|-7) !]^{s-1} \\
& \leq a_{2} H_{1}^{\alpha_{1}-2} H_{2}^{\alpha_{2}}[(|\alpha|-3) !]^{s} \sum_{\substack{0 \leq \beta \leq \alpha \\
4 \leq|\beta| \leq|\alpha|-3}} \frac{|\beta|^{4}(|\alpha|-|\beta|)^{3}}{} \\
& \leq a_{3} H_{1}^{\alpha_{1}-2} H_{2}^{\alpha_{2}}[(|\alpha|-3) !]^{s},{ }^{s}
\end{aligned}
$$

the last inequality following from Lemma 2.6. In view of the estimates for $I_{1}, I_{2}$ and $I_{3}$, we have, for any $\alpha \leq \alpha_{0}$ with $|\alpha| \geq 3$,

$$
\left\|\partial^{\alpha}(u v)\right\| \leq a_{4}\left(\|u\|_{3}+\|v\|_{2}+1\right)^{2} H_{1}^{\alpha_{1}-2} H_{2}^{\alpha_{2}}[(|\alpha|-3) !]^{s}
$$

with $a_{4}=a_{1}+a_{3}$. Thus the conclusion (臣) follows if we choose $\tilde{c}_{*} \geq a_{4}\left(\|u\|_{3}+\|v\|_{2}+1\right)^{2}$.

\section{Regularity of stratified water waves without surface tension}

Without surface tension, that is, when $\sigma=0$, the flow is driven only by the gravity. Then the equation (3b) becomes

$$
|\nabla \psi|^{2}+2 g(y+d) \rho=Q, \quad y=\eta(x) .
$$

Correspondingly, the equation (4b) becomes

$$
1+h_{q}^{2}+(2 g \rho h-Q) h_{p}^{2}=0, \quad \text { on } \quad p=0 .
$$

Proven in this section is the regularity property of all the streamlines and the pseudo-stream function of stratified water waves without surface tension.

Theorem 5.1. Consider the free boundary problem (3a)-(3d) with (3b) replaced by the above (3b'). Suppose $\beta \in C^{0, \mu}\left(\left[p_{0}, 0\right]\right)$ and $\rho \in C^{1, \mu}\left(\left[p_{0}, 0\right]\right)$ with $\mu$ and $p_{0}$ given. Then each streamline including the free surface $y=\eta(x)$ is a real-analytic curve. If, in addition, $\beta, \rho \in G^{s}\left(\left[p_{0}, 0\right]\right)$ with $s \geq 1$, then $\psi(x, y) \in G^{s}(\bar{\Omega})$; in particular if $s=1$, i.e., $\beta$ and $\rho$ are analytic in $\left[p_{0}, 0\right]$, then the pseudo-stream function $\psi(x, y)$ is analytic in $\bar{\Omega}$.

Proof. As before we only prove the corresponding regularity for height function $h$ of the system (4a) - (4c) with (4b) replaced by the above (4b'). Since the arguments are nearly the same as those 
in presence of surface tension (Section 3 and Section 44), we shall only give a sketch and indicate how to modify the analysis.

Repeating the arguments in Section 4, we can derive the second statement in Theorem 5.1. without any difference. So we only need to focus on the proof of the first statement on the analyticity of streamlines, which, as we will see below, is in fact simpler than its counterpart in Section 3 ,

As in Remark 3.3 we may assume $\partial_{q}^{k} h \in C^{2, \mu}(\bar{R})$ for any $k \in \mathbb{N}$. Taking $m^{\text {th }}$-order derivative with respect to the $q$-variable on both sides of the equation (4b') shows that the second equation in (11) becomes

$$
\tilde{B}(h)\left[\partial_{q}^{m} h\right]=\tilde{\varphi}_{1}+\tilde{\varphi}_{2}, \quad \text { on } p=0,
$$

with the operator

$$
\tilde{B}(h)[\phi]=h_{q} \phi_{q}+(2 g \rho h-Q) h_{p} \phi_{p}+2 g h_{p}^{2} \phi
$$

and the right-hand side

$$
\begin{gathered}
\tilde{\varphi}_{1}=-\frac{1}{2} \sum_{1 \leq n \leq m-1}\left(\begin{array}{c}
m \\
n
\end{array}\right)\left(\partial_{q}^{n} h_{q}\right)\left(\partial_{q}^{m-n} h_{q}\right)-\frac{1}{2}(2 g \rho h-Q) \sum_{1 \leq n \leq m-1}\left(\begin{array}{c}
m \\
n
\end{array}\right)\left(\partial_{q}^{n} h_{p}\right)\left(\partial_{q}^{m-n} h_{p}\right), \\
\tilde{\varphi}_{2}=-\rho g \sum_{1 \leq n \leq m-1}\left(\begin{array}{c}
m \\
n
\end{array}\right)\left(\partial_{q}^{n} h\right)\left(\partial_{q}^{m-n} h_{p}^{2}\right) .
\end{gathered}
$$

The first and third equations in (11) remain unchanged. Then, as before, our aim is to show that the corresponding estimate as in Proposition 3.1 holds, that is, there exists a constant $\tilde{L} \geq 1$ such that for any $m \geq 2$,

$$
\left(\tilde{E}_{m}\right): \quad\left\|\partial_{q}^{m} h\right\|_{2, \mu} \leq \tilde{L}^{m-1}(m-2) !,
$$

and to this end the main point is to show that $\left(\tilde{E}_{m}\right)$ holds under the assumption that for any $j$ with $2 \leq j \leq m-1$, the following estimates

$$
\left(\tilde{E}_{j}\right): \quad\left\|\partial_{q}^{j} h\right\|_{2, \mu} \leq \tilde{L}^{j-1}(j-2) !
$$

are already valid.

Since $h \in C^{2, \mu}(\bar{R})$, the coefficients of the operator $\tilde{B}(h)$ are in $C^{1, \mu}(\bar{R})$. Moreover by the induction assumption (171), $\tilde{\varphi}_{1}$ and $\tilde{\varphi}_{2}$ are in $C^{1, \mu}(\bar{R})$. Furthermore in this case the operator $\tilde{B}(h)$ satisfies the complementing condition in the sense of [1] since the coefficient $(2 g h-Q) h_{p}$ of $\phi_{p}$ is nonzero and satisfies

$$
(2 g \rho h-Q) h_{p}=\frac{1+h_{q}^{2}}{h_{p}} \geq \frac{1}{\sup _{\bar{R}} h_{p}} \geq \delta
$$

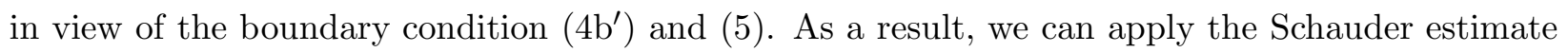
(see for instance [7, Theorem 6.30] and [1]) to conclude

$$
\left\|\partial_{q}^{m} h\right\|_{2, \mu} \leq \tilde{\mathrm{C}}\left(\left\|\partial_{q}^{m} h\right\|_{0}+\sum_{i=1}^{2}\left\|f_{i}\right\|_{0, \mu}+\sum_{i=1}^{2}\left\|\tilde{\varphi}_{i}\right\|_{1, \mu}\right),
$$


with $\tilde{\mathcal{C}}$ a constant independent of $m$, and $f_{i}, i=1,2$, defined in (12)-(13). Then we can use the similar arguments as in Section 3 without any additional difficulty, to conclude

$$
\tilde{\mathcal{C}}\left(\left\|\partial_{q}^{m} h\right\|_{0}+\sum_{i=1}^{2}\left\|f_{i}\right\|_{0, \mu}+\sum_{i=1}^{2}\left\|\tilde{\varphi}_{i}\right\|_{1, \mu}\right) \leq \tilde{C}_{1} \tilde{L}^{m-2}(m-2) !,
$$

with $\tilde{C}_{1}$ a constant independent of $m$. Choosing $\tilde{L} \geq \tilde{C}_{1}$, we complete the proof of the proposition.

\section{Acknowledgements}

The support of Foundation of WUST and support of Hubei Province Key Laboratory of SSMP are gratefully acknowledged.

\section{References}

[1] S. Agmon, A. Douglis, and L. Nirenberg. Estimates near the boundary for solutions of elliptic partial differential equations satisfying general boundary conditions. I, Comm. Pure Appl. Math., 12 (1959) 623-727.

[2] H. Chen, W.-X. Li and C.-J. Xu. Gevrey regularity of subelliptic Monge-Ampère equations in the plane. Advances in Mathematics , 228 (2011) 1816-1841.

[3] Adrian Constantin and Joachim Escher. Analyticity of periodic traveling free surface water waves with vorticity, Ann. of Math. (2), 173(1) (2011) 559-568.

[4] A. Constantin and W. Strauss. Exact steady periodic water waves with vorticity, Comm. Pure Appl. Math., 57 (2004), 481-527.

[5] J. Escher. Regularity of rotational traveling water waves, preprint.

[6] J. Escher, A.-V. Matioc and B.-V. Matioc. On stratified steady periodic water waves with linear density distribution and stagnation points, to appear in J. Differential Equations.

[7] D. Gilbarg and Neil S. Trudinger. Elliptic partial differential equations of second order. Classics in Mathematics. Springer-Verlag, Berlin, 2001. Reprint of the 1998 edition.

[8] Vera Mikyoung Hur. Analyticity of Rotational Flows Beneath Solitary Water Waves, Int Math Res Notices 2011., doi: 10.1093/imrn/rnr123.

[9] D. Henry. Analyticity of the streamlines for periodic travelling free surface capillary-gravity water waves with vorticity, SIAM J. Math. Anal., 42(6) (2011) 3103-3111.

[10] D. Henry. Analyticity of the Free Surface for Periodic Travelling Capillary-Gravity Water Waves with Vorticity, Journal of Math. Fluid Mech., 2011, DOI: 10.1007/s00021-011-0056-z.

[11] D. Henry. Regularity for steady periodic capillary water waves with vorticity, Phil. Trans. Roy. Soc. A, to appear. 
[12] D. Henry and B. Matioc. On the existence of steady periodic capillary-gravity stratified water waves, Ann. Scuola Norm. Sup. Pisa, to appear.

[13] D. Henry and B. Matioc. On the regularity of steady periodic stratified water waves, Comm. Pure Appl. Anal., to appear.

[14] D. Kinderlehrer, L. Nirenberg, and J. Spruck. Regularity in elliptic free boundary problems, J. Analyse Math., 34 (1978) 86-119 (1979).

[15] H. Lewy. A note on harmonic functions and a hydrodynamical application, Proc. Amer. Math. Soc., 3 (1952)111-113.

[16] W.-X. Li and L.-J. Wang. Regularity of traveling free surface water waves with vorticity, http://arxiv.org/abs/1112.2730.

[17] R. R. Long. Some aspects of the flow of stratified fluids. Part I : A theoretical investigation, Tellus 5 (1953), 42-57.

[18] B.-V. Matioc. Analyticity of the Streamlines for Periodic Traveling Water Waves with Bounded Vorticity, Int Math Res Notices, 17 (2011), 3858-3871. doi: 10.1093/imrn/rnq235

[19] B.-V. Matioc. On the regularity of deep-water waves with general vorticity distributions, to appear in Quart. Appl. Math., 2011.

[20] Luigi Rodino. Linear partial differential operators in Gevrey spaces. World Scientific Publishing Co. Inc., River Edge, NJ, 1993.

[21] R. E. L. Turner. Internal waves in fluids with rapidly varying density, Ann. Suola Norm. Sup. PisaCl. Si., 8 (1981), 513-573.

[22] S. Walsh. Stratified steady periodic water waves, SIAM J. Math. Anal., 41 (2009), 1054-1105.

[23] S. Walsh. Steady periodic gravity waves with surface tension, arXiv:0911.1375, 2009.

[24] C.-S. Yih. Exact solutions for steady two-dimensional flow of a stratified fluid, J. Fluid Mech., 9 (1960), 161-174. 